\title{
La molteplicità e la crisi
}

Multiplicity and crisis

\section{Stefania Consigliere e Cristina Zavaroni}

\section{OpenEdition}

\section{Journals}

\section{Edizione digitale}

URL: http://journals.openedition.org/aam/393

DOI: $10.4000 /$ aam 393

ISSN: 2038-3215

\section{Editore}

Dipartimento Culture e Società - Università di Palermo

\section{Notizia bibliografica digitale}

Stefania Consigliere e Cristina Zavaroni, « La molteplicità e la crisi », Archivio antropologico mediterraneo [Online], Anno XXI, n. 20 (1) | 2018, online dal 30 juin 2018, consultato il 10 décembre 2020. URL : http://journals.openedition.org/aam/393 ; DOI : https://doi.org/10.4000/aam.393

Questo documento è stato generato automaticamente il 10 décembre 2020.

\section{(c) (i) (9)}

Archivio antropologico mediterraneo è distribuita con Licenza Creative Commons Attribuzione - Non commerciale - Non opere derivate 4.0 Internazionale. 


\title{
La molteplicità e la crisi
}

\author{
Multiplicity and crisis
}

\section{Stefania Consigliere e Cristina Zavaroni}

War, slavery, captivity, authoritarianism, the theft of culture and of the means for creating autonomous, sustainable life, the attachment to epistemologies of blindness, and the investment in ontologies of disassociation remain the key problems of our time.

Avery F. Gordon

Ai nostri amici: alle donne e agli uomini konzo. E a Francesco Remotti e ai suoi, dall'altra parte della montagna.

\section{La teoria necessaria ${ }^{1}$}

\section{Teoria dal campo}

1 Ineludibile punto di partenza della teoria antropologica, la centralità del campo è oggi anche luogo prospettico da cui pensare le questioni operative e politiche che si aprono all'intersezione fra discipline e nell'interazione fra mondi (Viveiros de Castro 2002; Da Col, Graeber 2011; Graeber 2015; Holbraad, Pedersen 2017). Via regia per la «decolonizzazione del nostro pensiero», la theory from the field è fra gli strumenti più promettenti, ancorché perturbanti, nel confronto con la crisi globale in corso (Stengers 2009; Danowski, Viveiros de Castro 2014): un hopeful monster alla cui ombra quest'articolo si apre.

2 Tradizionalmente situato "lontano da casa" e poi arrivato, in intreccio con la sociologia, "vicino a casa", oggi il campo si apre anche "sotto casa" in una forma completamente diversa e tutto sommato ancor più critica, in quanto portatrice di un'urgenza pressante e dagli esiti incerti. Parliamo, naturalmente, dei flussi migratori e in particolare di quelli forzati, indotti da guerre, da violenze di vario genere e dall'impossibilità di 
proiettarsi in un orizzonte esistenziale locale ${ }^{2}$; e del loro impatto sulla vita sociale e politica italiana e sui soggetti chiamati a intervenire.

3 Se della parola "campo" facciamo risuonare tutte le accezioni, incluse quelle più sgradevoli, possiamo dire che esiste oggi, all'interno dei nostri confini nazionali, un enorme campo dai confini mobili, infinitamente frastagliato, nel quale si concentrano umani, investimenti simbolici e materiali, biopolitiche, e un coacervo di azioni militari, mediche, legislative, socio-assistenziali, umanitarie, educative (Baubet, Moro 2009; Rastello 2010). Esso si configura come uno spazio antropologico del tutto peculiare, interamente strutturato sulla precarietà, ovvero sulla «condizione di essere vulnerabile agli altri» (Tsing 2015: 20): da un lato coloro che migrano, portatori di forme umane inedite e spesso "aperte" - nel senso di "violate" - e a rischio di crisi, plasmate dal contesto di provenienza, dal tragitto migratorio, dall'esperienza del trattenimento (Buffa 2015) e dall'attrito con la società ospitante (Bäärnhielm et al. 2017); dall'altro coloro che accolgono, reclutati da un enorme bacino di disoccupazione, impreparati allo spaesamento che l'alterità induce e destinati a un avvicendamento rapidissimo dettato dal burnout; al di sopra di tutto un impianto legislativo caotico a livello nazionale e opaco a livello internazionale, un vero e proprio spazio d'eccezione giuridica (Agamben 2003) che funziona soprattutto come dispositivo di messa in forma dei migranti a uso della società ospitante (Zorzetto 2016). Qui ha luogo l'incontro selvaggio - e cioè privo di spazi e strumenti che ne contengano la forza perturbante - di cosmovisioni esogene e dei fantasmi che le popolano ${ }^{3}$.

Tutto ciò non solo interroga ma anche sfida lo sguardo antropologico, ponendo questioni che richiedono la capacità di muoversi ai suoi limiti in relazione con altre prospettive teoriche (psicologia, psichiatria, pedagogia, sociologia, storia); di cogliere nella loro connessione le crisi che si manifestano fra migranti e operatori; di rispettare i saperi esogeni che s'incontrano oggi sulle nostre strade e che difficilmente possono manifestarsi iuxta propria principia (Beneduce 1998); e di farsi sensibili a ciò che delle strutturazioni antropopoietiche resta in ombra, destinato a essere poco dicibile o del tutto invisibile. A partire da qui occorre pensare, perché in queste circostanze il sapere antropologico si trova fin da subito non solo a intervenire, ma anche, fatalmente, a prendere posizione.

\section{La domanda}

5 È un campo che entrambe abbiamo praticato in diversi modi lungo gli ultimi quindici anni: dalle formazioni per gli enti territoriali alla mediazione scolastica e nei servizi, dalla partecipazione a sedute etnocliniche alle consulenze antropologiche per equipe di psicologi, psichiatri, educatori, assistenti sociali ecc. Che si tratti di SPRAR, di CAS, di scuole, di ambulatori, di sostegno psicologico o di intervento sociale, e nonostante l'attenta sensibilità di molti operatori, ciò che riguarda la popolazione migrante è spesso avvertito e gestito all'insegna dell'emergenza. Accuratamente preparata dall'intera filiera della migrazione e poi dal discorso pubblico, l'urgenza inibisce ogni più ampia riflessione storica e sociale sulle cause dell'emergenza, sulle articolazioni fra geopolitica e vita psichica, sulla necessità di spazi diplomatici fra mondi. La naturalizzazione dei ruoli e delle posizioni occulta la violenza strutturale che regola i processi migratori, rimuovendola sotto etichette, come quella di PTSD, che rimandano a malfunzionamenti psichici individuali anziché a dinamiche politiche globali (Bergner 2009: 323) e comprimendo i soggetti in ruoli burocraticamente imposti ("richiedente", 
"rifugiato", “operatore", "responsabile") che rinforzano le gerarchie implicite dell'ordine mondiale postcoloniale. È frequente lo scivolamento semantico - che tradisce uno scivolamento etico - da "operatore dell'accoglienza" a "educatore", da "psicologo" a "valutatore", da "richiedente/rifugiato/migrante" a "ragazzo": come se coloro che arrivano non disponessero già di una soggettività piena e di un modo efficace di abitare il mondo, ma fossero pensabili solo come soggetti dimidiati, materialmente e culturalmente deprivati; oppure come vittime. L'intrinseca superiorità del nostro modo - italiano, euro-americano, occidentale, moderno - di fare umani e di abitare il mondo è lo sfondo impensato di tutte le azioni.

6 Le situazioni più acute s'incontrano nell'ambito del cosiddetto "sistema dell'accoglienza", ma l'impianto emergenziale/rieducativo resta in azione anche quando, superata l'accoglienza, la popolazione in arrivo si confronta con gli istituti di quella ospitante, facendone ripetutamente inceppare gli automatismi. Gli operatori si trovano allora a constatare lo scacco delle loro azioni e delle loro buone intenzioni: lo sconcerto a fronte di soggetti, dinamiche e crisi che sentono di non capire li porta a volte a prender distanza dall'urgenza e a domandarsi infine chi siano coloro che hanno di fronte, e come accompagnarli nel loro transito. Qui talvolta entrano in scena gli antropologi, chiamati nella speranza che possano fornire una sorta di bignamino culturalista o, peggio, che dispongano di un accesso privilegiato alla "verità" dei migranti e della loro storia. Aspettativa che, puntualmente, andrà decostruita, evitando però di smarrirne il senso profondo: quello di un'apertura possibile alla pluralità.

7 Lo sconcerto degli operatori è il varco per l'apertura di spazi capaci di far coesistere, almeno provvisoriamente, mondi differenti, dove sia possibile osservare la molteplicità delle forme di umanità, delle cosmovisioni, dei modi della crisi e delle piste di reintegro, e costruire nuove narrazioni: temi classici dell'antropologia, della mediazione e della clinica etnopsichiatrica, che si ripresentano ogni volta che lo sfondo etnocentrico vacilla (Devereux 1967; Nathan, Stengers 1996; Bouznah, Lewertowsky 2017). In queste circostanze la presenza degli antropologi è utile non solo (e non tanto) per le conoscenze etnografiche puntuali di cui dispongono, quanto per il tipo di sguardo di cui sono portatori: quello che presuppone la differenza, l'opacità, la complessità e la profondità dei mondi umani e dei loro rappresentanti; consapevole che la proiezione, l'equivoco e l'incomprensione sono passaggi indispensabili nell'incontro fra alterità (De Pury 2005; Bouznah, Lewertowski 2017); e disposto a dare voce al fantasma: non l'invisibile o l'ineffabile, ma uno

stato di animazione in cui una violenza sociale repressa o irrisolta si rende nota, a volte in modo molto diretto, a volte in modo più obliquo [...] l'apparizione di spettri o fantasmi ci avverte che ciò che è stato nascosto è ancora molto vivo e presente $\mathrm{e}$ interferisce proprio con quelle forme, sempre incomplete, di contenimento e repressione che incessantemente subiamo (Gordon 1997: xvi).

Le ambiguità del potere, l'angoscia, la violenza geopolitica, i diversi modi di essere persona, i legami insospettati, le speranze divergenti, le ombre della storia e le paure del presente: tutto questo emerge nell'incontro fra alterità, molto al di là delle buone intenzioni, in un "campo" che risulta ignoto a tutti gli attori coinvolti (Marcus, Fischer 1999). 


\section{Uno sfondo noto?}

Quando Devereux elaborava il metodo etnopsichiatrico poteva contare sul fatto che gli sfondi sui quali leggere i pazienti fossero noti e affidabili (Devereux 2007; Delille 2016). Non solo quello, supposto universale, della psicoanalisi, chiamata a far luce sulla parte in ombra dei fenomeni umani, ma anche quelli locali descritti dalle etnografie: un paziente mohave poteva essere compreso attraverso le etnografie sui Mohave. E di fatto, fino a qualche decennio fa, gli antropologi potevano studiare le società umane "come se" fossero stabili, disponessero di confini che ne segnavano in maniera univoca un dentro e un fuori e mettessero in forma i propri membri in modo abbastanza uniforme (Falk Moore 1994; Arce, Long 2000).

Poi questa lavorazione della diversità culturale, che leggeva le culture come contenitori coerenti, discreti e più o meno stabili, è andata in frantumi. Sul fronte interno le evoluzioni disciplinari hanno promosso una visione molto più conflittuale, instabile $\mathrm{e}$ processuale sia delle culture che dei soggetti che ne fanno parte. Sul fronte esterno, il definitivo estendersi della logica economica e antropologica euro-statunitense a scapito delle logiche locali, che hanno progressivamente perduto parte della loro riconoscibilità, trasmissibilità e tenuta, ha letteralmente imposto la questione del meticciato.

11 La possibilità (o la necessità) dell'ibridazione si è posta quasi ovunque in termini piuttosto ripetitivi: nella maggior parte dei casi si è trattato di scendere a patti con la cosmovisione veicolata dai mercati, dallo stato, dalla scienza, dalla biomedicina, dalla scolarizzazione; e con la violenza strutturale che la media. Un processo che sembra raggiungere un nuovo culmine nei nostri anni e che produce, fra l'altro, anche il massiccio fenomeno migratorio che abbiamo sott'occhio (Beneduce 2016). Ma a differenza di quanto accadeva all'epoca di Devereux, oggi il confronto fra alterità avviene su uno sfondo molto più incerto. Le etnografie non dicono più molto sulla strutturazione di coloro che, essendo nati in un contesto, sono poi migrati: un po' perché gli istituti culturali locali sono quasi ovunque indeboliti, resi fragili dall'estensione dei mercati e dallo statuto subalterno in cui il processo di modernizzazione li fa scivolare; un altro po' perché spesso gli attuali percorsi migratori sono, di per sé, fortemente traumatici ed effettuano una deculturazione più o meno violenta di coloro che li percorrono ${ }^{4}$. L'effetto perturbante dei migranti sulla coscienza dei "paesi ospiti" dipende anche dal fatto che illuminano, nella loro stessa esistenza, il rimosso coloniale e geopolitico che governa il discorso pubblico.

12 Molto di ciò che oggi avviene non si sviluppa, se non marginalmente, per una dinamica interna ai gruppi coinvolti, ma è l'esito della violenza delle relazioni coloniali e postcoloniali e dell'espansione dei mercati neoliberisti. Su questo sfondo conflittuale occorre leggere i processi culturali e le traiettorie biografiche, in un contesto globale dove le piste di soggettivazione che fanno esplodere l'antica nozione di soggetto sono anche quelle di nuovi e crudeli assoggettamenti e dove le modalità creative di negoziazione culturale sono rese indispensabili dall'imposizione manu militari di una logica esogena ${ }^{5}$. L'impresa è delicata: si naviga a vista fra lo scoglio del pessimismo critico e quello, altrettanto letale, del "politicamente corretto", che enfatizza la fluidità dei soggetti e dei gruppi a scapito della permanenza e del valore dei loro attaccamenti (v. sotto), finendo col riproporre un'idea di umano - libero, autonomo, inventivo, 
plastico, alla ricerca del proprio utile - tanto etnocentrica e conforme al modello egemone quanto inconsapevole (Sahlins 2014).

Qui tenteremo un'altra via, più lunga, che passa per il mare aperto dell'immaginario e prova a mettere in tensione due campi in cerca dei luoghi dove le logiche subalterne, anziché essere sussunte da quella moderna, continuano ad agire producendo dentro la modernità spazi a essa discontinui che prevedono la possibilità del molteplice ${ }^{6}$. Possibilità che non è già data in partenza: «La coesistenza, in sé e nel mondo, di eterogeneità multiple (umane, religiose, politiche e culturali) non va da sé. Dev'essere costruita» (Sironi 2017: 19).

\section{Un modello operativo} indispensabile in tutte le situzioni in cui h teoria deve orientare una protica, decostruendo la presunzione di superiorità che sottende l'assetto moderno ${ }^{7}$. Nei diversi contesti operativi che abbiamo praticato, ci è stato utile declinare la teoria dell'antropopoiesi proposta da Remotti $(2002,2011)$ in termini di attaccamenti, per come questi sono definiti e impiegati in etnopsichiatria: una varietà di connessioni, scambi, vincoli, che quasi letteralmente "tengono" il soggetto nel mondo attraverso un fascio di relazioni che sono fin dall'inizio costitutive (Latour 2000a; Nathan 2003; Coppo 2013). Che siano già dati o da instaurare, consapevoli o invisibili, gli attaccamenti sono sempre riconosciuti e lavorati all'interno di una particolare organizzazione materiale e immateriale dell'esistente: abbastanza specifica da essere riconoscibile, abbastanza permanente da essere trasmissibile e abbastanza strutturata da disporre di una sua logica (Singleton 2017). Si potrebbe parlare di "logiche antropologiche" - a patto d'intendere, nella parola logica, più un sistema di rimandi armonici e di disarmonie sistemiche che una connessione di ordine matematico; e, soprattutto, a patto di non limitarla a ciò che è esplicito, fisso e oggettivabile, ma di pensarla come strutturazione, sempre provvisoria, di un modo di vivere e di sentire, dove non c'è soluzione di continuità fra le formazioni culturali e sociali (che siano consapevoli e descrivibili oppure implicite) e la presenza vivente dei soggetti, le strutture emotive e i modi cognitivi da cui sono attraversati e che attraversano ${ }^{8}$.

Non tutto della messa in forma culturale è conoscibile o dicibile: la parte visibile degli attaccamenti sprofonda nella zona in ombra dell'immaginario. Esistono diversi livelli di consapevolezza antropopoietica (Remotti 2002) e nella memoria non ereditaria di ciascun gruppo ci sono zone non consapevoli, poco lavorate, o addirittura rimosse e indicibili; campi di tensioni e contraddizioni, spinte centrifughe, anomalie e conflitti dove la storia dei gruppi e la biografia dei singoli incidono memorie, traumi, nostalgie e interdetti dalla natura e dalla temporalità difformi (Gordon 1997; Tsing 2015). Ciò significa che è possibile leggere in modo storico, contestuale e relazionale non solo $\mathrm{i}$ fenomeni relativi alla presenza al mondo (de Martino 1977), ma anche quelli relativi 
alla crisi, cercando in ciò che è percepibile tracce di ciò che è invisibile, nel vacillare del singolo l'impronta del gruppo e dello stato del mondo (Kleinmann 1980, 1995; ScheperHughes, Lock 1987; Good 1999; Baer et al. 1997): un processo che prende il nome di "funzione patoplastica" della cultura (Shorter 1992; Nathan 2003; Coppo 2003).

È nel confronto, sempre incerto, con questa sfera immaginaria, con le crisi che vi si aprono e con i fantasmi che la popolano, che le logiche culturali si riproducono, si riprendono e si approfondiscono. Questa è anche la zona umbratile dove è necessario avventurarsi quando il controtransfert culturale - ciò che, nella «somma totale delle distorsioni della percezione e delle reazioni» verso l'altro dipende da nuclei inconsapevoli determinati dalle proprie appartenenze culturali (Devereux 1967: 98) rischia di arrestare i processi biografici e culturali, bloccando soggetti e gruppi nella gabbia di ciò che la propria cultura ha pre-giudicato.

\section{Crocevia}

18 Fantasmatico è, ai nostri occhi, parte di ciò che i migranti portano con sé: gli spiriti che vengono a disturbarli; le volontà degli antenati; le ragioni prime e ultime della migrazione; i traumi storici; i mandati familiari che li vincolano; i riscatti frontalieri pagati con manodopera, delinquenza o prostituzione; la detenzione e le tradotte; la filiera della tratta; i viaggi di sete, fame, deserto e mare; a volte la tortura. Racconti difficili da ascoltare per gli operatori: un po' per la violenza non riscattabile del loro contenuto; un altro po' perché vi trapela uno "stato del mondo" che spesso, nelle nazioni prime, può arrivare solo in forma offuscata e censurata; e infine per via delle entità e delle forze che quei discorsi convocano, quella molteplicità di mondi umani che, tipicamente, espone i cittadini dei paesi ospitanti a un difficile spaesamento culturale.

19 Ma almeno altrettanto fantasmatici sono il dispositivo dell'accoglienza, dove l'epistemic murk descritto da Taussig (1987) - l'impossibilità strutturale di separare con una demarcazione sicura il vero dal falso, ciò che esiste da ciò che non esiste, quello che può essere provato da ciò che è "solo" narrazione - assume caratteri che, per essere grotteschi, non sono meno dannosi'; e quello più ampio del paese ospitante, fatto di individui che si pensano autonomi, di feticismo della merce, di sussistenza salariale; di disincanto sbandierato e, nonostante la gravità della crisi globale, di una solidissima fiducia etnocentrica nei propri criteri ontologici, epistemologici ed etici. Costruita sopra un assunto aggressivamente monista, la cosmovisione euro-americana è spesso incapace di immaginare forme di umanità differenti dalla propria, se non squalificandole come selvagge, barbare o terroristiche. Da qui la necessità di allestire spazi dove visioni del mondo radicalmente dissimili possano incrociarsi senza troppa violenza; e la loro urgenza: per via di quel che accade ai migranti, in primo luogo, e dell'insostenibilità di questa nuova tratta; ma anche per via di quanto accade a chi accoglie, messo di fronte, senza preparazione, a una vera e propria "guerra dei mondi» a bassa intensità (Latour 2000b).

Le storie di migranti che arrivano alla clinica etnopsicologica o alla consulenza antropologica sollevano una miriade di quesiti. Ciascuna va esplorata secondo le sue linee, contestualizzandola in una storia biografica e di gruppo, immergendosi negli impliciti che la animano e studiando pazientemente come questi entrino in relazione con quelli della cultura ospitante. Inevitabile una certa dose d'azzardo: la clinica 
etnopsichiatrica ha mostrato da tempo che la lavorazione degli attaccamenti - quelli dei migranti come quelli degli operatori, i fantasmi degli uni e gli spettri degli altri impone un'ineludibile provvisorietà epistemologica (Beneduce 1998; Sironi 2017). Alcune domande, tuttavia, si ripresentano ogni volta, come se fossero il precipitato di tutte le questioni specifiche, il loro aggregato: di quali gruppi sono rappresentanti le persone che incontriamo? Che tipo di esistenza hanno gli attaccamenti che portano? Che forma prendono gli umani nei contesti dove la logica della modernità si sovrappone a una logica locale indebolita? Domande che ammettono l'evenienza della pluralità, senza ancora assumerla. Qui emerge anche la questione decisiva attorno a cui s'incentra quest'articolo: la compresenza in uno stesso contesto o in un medesimo soggetto di logiche e modelli differenti è di per sé un fattore di rischio (tende, cioè, a generare più crisi, o crisi più profonde, rispetto a contesti monoculturali), oppure le crisi dipendono dall'attrito specifico prodotto dalle diverse logiche? Oppure ancora, la pluralità di modelli rappresenta un fattore protettivo in virtù della disponibilità di maggiori risorse interpretative?

\section{L'ignoto e l'analogia}

21 Laddove la trama dei processi e dei contesti che fanno da sfondo ai fenomeni sia poco conoscibile, e dove il "normale buon senso" rischi di portare fuori strada, l'ultima strategia conoscitiva che resta a disposizione è quella analogica, con tutta l'alea che il ragionamento sintomatico porta con sé (Melandri 2004; Ginzburg 1979) ma, anche, con la protezione che esso offre dai rischi connessi alle derive identitarie (Remotti 2013). Si tratta dunque di provare a mettere in rapporto fenomeni che potrebbero illuminarsi reciprocamente in luoghi dove la luce diretta è raramente disponibile, distaccandosi dall'emergenza, dal disincanto, dall'assimilazione, dalle diagnosi e dall'ansia di riportare subito l'ignoto al già noto.

Per noi l'uso dell'analogia ha spesso significato mettere in tensione elementi del "campo vicino" con altri situati in un "campo lontano" (e, in particolare, quello che conosciamo in modo più approfondito): spostando l'attenzione verso un altrove abbastanza distante - e placando quindi, per un momento, il senso di urgenza - la triangolazione consente di mettere meglio a fuoco ciò che, osservato da un unico punto di vista, è meno visibile.

23 I Bakonzo - un gruppo bantu di circa 600.000 persone stanziato sul massiccio del Rwenzori, in Uganda - hanno accolto una di noi (CZ) fra il 2004 e il 2017, durante otto diverse missioni di ricerca per un totale di circa due anni di permanenza. L'altra (SC) è stata loro ospite in occasione della missione del 2009-10. Molti temi specifici d'indagine si sono susseguiti nel corso degli anni ${ }^{10} \mathrm{e}$, nel suo complesso, il materiale raccolto configura un'etnografia abbastanza completa, dalla quale trarremo gran parte delle informazioni riportate nei prossimi paragrafi. A volte essa conferma o integra quanto già presente nella succinta letteratura disponibile; altre volte, invece, disconferma le informazioni riportate da altri ricercatori.

Quanto va accadendo fra i Bakonzo - dove scolarità è sinonimo di modernità e dove l'adesione ai grandi monoteismi ha rappresentato, per coloro che oggi sono adulti, una conversione esistenziale tra un prima e un dopo spiritualmente qualificati in modi differenti (Zavaroni 2008a) - è, per le istanze che muovono questa ricerca, esemplare: da un lato permette di porre in modo relativamente più semplice alcune domande che 
emergono anche nel contesto migratorio; dall'altro evidenzia come la pluralità, lungi dall'essere di per sé un fattore di crisi, può essere una risorsa terapeutica, nonché la garanzia di una possibilità allargata di presenza.

Quest'articolo intende pertanto essere un contributo alla riflessione in corso, fra antropologia e scienze della psiche, su quel che accade nelle situazioni in cui gli umani prendono forma all'intersezione fra logiche differenti, sulle crisi che in questi contesti possono aprirsi e sulle piste della loro risoluzione. Un breve quadro storico ed etnografico tratteggerà il presente dei Bakonzo in relazione alla loro storia recente, illustrando la compresenza di diversi insiemi di attaccamenti, rispondenti a logiche antropopoietiche e terapeutiche piuttosto eterogenee; tre storie di crisi lasceranno emergere ciò che accade quando i diversi attaccamenti entrano in conflitto ${ }^{11}$; per finire, andremo alla ricerca, tramite confronto analogico, di alcune delle dinamiche strutturali presenti in entrambi campi, ciò che porterà a un'ipotesi di coesistenza fra logiche.

\section{Spiriti in guerra}

\section{Una regione remota}

A seconda delle stagioni e dello stato delle strade, i $371 \mathrm{~km}$ che separano Kampala da Kasese richiedono dalle cinque alle otto ore. Da Kasese, capoluogo dell'omonimo distretto, si arriva poi in poco meno di un'ora al villaggio di Ibanda, luogo principale di questa ricerca e ultimo importante insediamento sulla sterrata che porta all'ingresso del Rwenzori Mountain National Park ${ }^{12}$. Il suo territorio si estende sul versante sud del fiume Mubuku, una valle fertile e ricca d'acqua dove, dal 1953, è presente una centrale idroelettrica.

Al confine con la Repubblica Democratica del Congo, Ibanda e la regione di Kasese sono piuttosto remote rispetto alla capitale nazionale, ma tutto sommato, nel contesto ugandese, ben collegate. Non altrettanto si può dire di Bundibugyo ${ }^{13}$ e della sua regione, situati a ovest della catena del Rwenzori e quindi geologicamente appartenenti al bacino del Congo nell'Africa Centrale. I $37 \mathrm{~km}$ di sterrata di montagna che s'inerpicano su per il massiccio richiedono fino a tre ore e mezzo di trasporto $4 \times 4$ di grandi dimensioni. Sulla strada s'incrociano mezzi pesanti che portano verso i mercati esteri il the, il caffè e il cacao prodotti sul Rwenzori, nonché le merci di contrabbando scambiate tra Uganda e RDC. È un tragitto redditizio ma faticoso, e il governo ugandese non ha alcun interesse a rinnovare la rete stradale: gruppi di ribelli sono rifugiati nella foresta congolese dell'Ituri (Jourdan 2010) e l'asperità del percorso fa da cuscinetto fra le due nazioni.

Fra Kasese e Bundibugyo risiede larga parte dei Bakonzo ${ }^{14}$. A ovest del Rwenzori, in territorio congolese, sono stanziati i Banande studiati da Francesco Remotti (1993, 1994), che i Bakonzo percepiscono come "popolazione sorella", separata dal confine coloniale fra il territorio belga e quello inglese. Escludendo le recenti acquisizioni dai lessici europei, i due gruppi parlano una lingua del tutto sovrapponibile ed entrambi si riconoscono nell'etnonimo Bayira. Gran parte della popolazione Bakonzo, e soprattutto di quella più anziana, parla lhukonzo ${ }^{15} \mathrm{e}$ altre lingue locali utili per comunicare con le etnie circostanti (in particolare rutooro e luganda) e non parla l'inglese; tutti gli informatori di campo coinvolti in questa ricerca, per contro, parlano inglese correntemente. 
Ancora poco noti in letteratura (Alnaes 1967; Cooke, Doornbos 1982; Magezi et al. 2004; Pennaccini 2006; Pennacini, Wittenberg 2008; Facci 2009; Gardoncini 2009), i Bakonzo sono un gruppo etnico ${ }^{16}$ a cui si appartiene per ascendenza patrilineare e che si distingue da quelle circostanti per una serie di tratti caratteristici che, come vedremo, si sono andati riconfigurando sotto la pressione della storia coloniale e che, nel loro insieme, hanno una funzione strutturante: il sistema nominale (Zavaroni e Consigliere 2018); gli spiriti convocati e il loro habitat; le scarificazioni (esyonzole) e le acconciature fra le donne; la circoncisione (olhusumba) fra gli uomini. Quest'ultima è anche una delle caratteristiche per cui i Bakonzo sono noti al resto dell'Uganda (Alnaes 1967; Zavaroni 2008b), dove solo altri due gruppi circoncidono: i Baamba stanziati sulle colline a nord del Rwenzori e i Bagisu del Mount Elgon (Heald 1999; Khamalwa 2004). Fra i Bakonzo essa veniva praticata nel contesto di un rito iniziatico piuttosto difficile le cui stagioni, che già erano proclamate a intervalli irregolari, sono cessate del tutto una quarantina di anni fa.

La perifericità geografica e geologica del territorio konzo è un fattore importante della loro storia recente: non solo perché è connessa alle peculiarità e al curioso destino degli spiriti konzo, ma anche perché ha lungamente trattenuto l'incedere della modernità nelle sue diverse declinazioni (diffusione dei monoteismi, scolarizzazione, centralizzazione, regime salariale ecc.). Se è vero che il processo di modernizzazione, col controllo/produzione della popolazione che esso permette e richiede, dipende dalla "quantità di stato" (stateness, Scott 2002: 15) e si espande dal centro alla periferia e dalle classi più abbienti a quelle più marginali, allora diversi fenomeni dell'attuale società Bakonzo testimoniano di un'inclusione tardiva nelle sue dinamiche: dalla comparsa recente dei cognomi coniugali alla diffusione della scolarità, dalla compresenza non pacificata di spiriti e Spirito alla recentissima adozione di una figura di "re" sopra un impianto di distribuzione del potere tradizionalmente acefalo.

31 Ciò riguarda in special modo la zona di Bundibugyo che, sotto ogni profilo relativo alla "quantità di stato" (amministrazione, sanità, servizi, scuole, modi di sussistenza, infrastrutture, diffusione di beni di consumo e di mezzi di trasporto ecc.), ad eccezione della massiccia presenza militare, si pone in modo fortemente discontinuo rispetto alla regione di Kasese. I due distretti sono però, nell'immaginario konzo, complementari: i Bakonzo stanziati attorno a Kasese rintracciano le loro origini e le loro tradizioni a nord della montagna, mentre quelli di Bundibugyo trovano a sud del massiccio il riconoscimento sociale, economico e politico che manca nel loro territorio. Questa tensione generativa si è stabilita parallelamente a tre importanti fenomeni che, tra il periodo coloniale e quello postcoloniale, hanno ridefinito la vita materiale e spirituale dei Bakonzo: la creazione del Rwenzori Mountain National Park, la cessazione delle stagioni iniziatiche e le lotte politiche del movimento di ribellione indipendentista Rwenzururu.

\section{Peripezie di un regno}

32 Cominciamo da queste ultime e dalla peculiare dialettica che le conquiste del movimento Rwenzururu ha instaurato fra logica locale e logica coloniale. Fatto anomalo in un'area caratterizzata da grandi regni, i Bakonzo non hanno mai esperito un processo di centralizzazione, né c'è mai stato un governo di tutti i Bayira (Bakonzo più Banande). E mentre questi ultimi distribuivano le terre e raccoglievano le tasse 
secondo un sistema gestito da una complessa struttura di capi (mwami: Remotti 1982, 1994), fra i secondi, fino a tempi recenti, il potere era distribuito fra il sistema dei clan e quello, basato sulla sequenza degli insediamenti, dei crinali (Gardoncini 2009, 2010). A questi due sistemi si sono poi sovrapposti quello statale elettivo ${ }^{17}$ e quello del Regno Rwenzururu, nato dalle lotte del movimento omonimo, a capo del quale si trova l' Omusinga ("re").

L'importanza materiale e simbolica della lotta Rwenzururu si radica nella storia coloniale. All'arrivo degli inglesi nel 1888, i Bakonzo abitavano i territori montani fra i 1500 e i 3000 metri. Orticultori - ma non coltivatori, né allevatori - scendevano in pianura quasi solo per l'approvvigionamento di sale al lago Katwe e avevano scambi sporadici con gli altri gruppi. La nuova potenza creò alleanze con i principali regni del territorio ugandese e, nell'area del Rwenzori, stabilì il Protettorato a Fort Portal, capitale del regno Batooro, a cui fu affidato il controllo del territorio konzo. In un primo tempo la raccolta delle tasse per mano batooro spinse i Bakonzo ancora più alto sulla montagna; poi, quando nelle pianure verso sud-ovest furono avviate coltivazioni di cotone, caffè, mais e canna da zucchero, mano d'opera bakonzo scese a valle, ampliando l'area dei loro insediamenti.

I Bakonzo raccontano di aver subito, all'epoca, una duplice marginalizzazione: all'oppressione coloniale si sommava quella dei vicini Batooro, che ricoprivano tutte le cariche amministrative e che, in occasione delle visite degli ispettori, allontanavano $\mathrm{i}$ bambini konzo dalle scuole per nasconderli e privare i Bakonzo del rapporto diretto con la potenza coloniale. Così, fino alla fine degli anni '60 la lingua informale dell'amministrazione, del commercio e dell'istruzione rimase il rutooro e perfino le chiese cristiane raggiunsero la popolazione konzo solo attraverso i missionari tooro ${ }^{18}$.

Anche il rapporto con la lingua racconta questa storia di potere. L'emarginazione aveva persuaso i Bakonzo che il mantenimento della lingua e dei culti tradizionali fosse in parte responsabile della situazione, ciò che spinse molti ad abbandonarli. Il lhukonzo diventò la lingua dei retrogradi, di quelli che ancora non conoscevano i benefici dello stile di vita occidentale di cui gli oppressori Batooro erano gli involontari portatori. Per un bambino konzo degli anni ' 50 e ' 60 il primo passo verso l'emancipazione passava per l'apprendimento del rutooro nelle sub-grade schools, a cui seguiva l'apprendimento della lingua inglese nelle scuole primarie e superiori (Eickelman 1985; Erny 2001; Simpson $2009)^{19}$. A tutt'ora sono pochissimi i testi disponibili in lhukonzo e gli scolari konzo hanno risultati scolastici in lingua inglese più bassi rispetto al resto della popolazione dell'Uganda del sud ${ }^{20}$.

36 A modificare il rapporto dei Bakonzo con i loro istituti tradizionali - da ricettacolo di ciò che è da nascondere o superare in quanto simbolo di subalternità, ad asse di valorizzazione identitaria e di recupero affettivo e politico - sono state le peripezie del movimento Rwenzururu, che è oggi per i Bakonzo sinonimo di ricostruzione (o reinvenzione) dell'identità etnica e di battaglia per l'autonomia (Cooke, Doornbos 1982; Syahuka-Muhindo 1991, 2008; Stacey 2003). Inevitabile, qui, ripensare alla lunga discussione sulla classificazione etnica come «prodotto di processi storici che strutturano relazioni di diseguaglianza fra entità sociali discrete» (Comaroff, Comaroff 1992: 65; 1999).

Nato poco prima dell'indipendenza ugandese, sancita nel 1962, col programma di ottenere il riconoscimento di un regno konzo distinto e indipendente dal regno tooro, la sua storia è segnata da lotte sanguinose contro i Batooro, contro l'esercito nazionale 
e contro gruppi di oppositori konzo. Suo fondatore e leader indiscusso fu Isaya Mukirania e molti di coloro che oggi si definiscono rwenzururu hanno combattuto a più riprese nella foresta. La creazione del distretto di Kasese come costola secessionista del più ampio distretto tooro, avvenuta nel 1974 a opera del governo di Idi Amin, si situa nell'ambito di questa disputa (Stacey 2003; Baluku 2008; Pennacini 2006).

Il figlio di Isaya, Charles Wesley Mumbere, è oggi re dei Bakonzo. Nonostante la continua ambivalenza del governo nazionale nei confronti dei regni tradizionali (Gifford 1999), l'unica forma possibile di riconoscimento di un'istituzione tradizionale sembrava (e sembra) essere quella del regno: qui, pertanto, ha scelto di passare anche la società konzo, che pure era rimasta lungamente acefala. I risultati del movimento Rwenzururu possono dunque essere messi sotto un duplice segno: recupero sentimentale, in chiave identitaria, di ciò che è percepito far parte della tradizione e istituzione di un sistema di potere mimetico rispetto a quello degli oppressori ${ }^{21}$.

Un'ambiguità che si complica fino a un completo epistemic murk quando dalla politica pubblica e dagli eventi oggettivabili si scivola, senza alcuna vera soluzione di continuità, fino ai paesaggi remoti della crisi e della cura: «Il re dei Bakonzo ha detto di cominciare a fare le cose del passato, che così rivivranno»"22, afferma una donna che porta la sofferenza incisa fin nel nome e che sa, attraverso i sogni, che solo nel rivivere delle "cose del passato" si aprirebbe, per lei, un pista di guarigione.

\section{Fare adulti al tempo del dio unico}

Tra la zona di Bwamba ${ }^{23}$ e la valle del Mubuku i rapporti sono stretti: un sentiero, tutt'ora utilizzato, passa per le montagne e collega le due aree in un giorno di cammino. Il rito di circoncisione olhusumba si svolgeva appunto nella zona di Bwamba, dove gli iniziandi si recavano da tutte le parti del bukonzo: diventare uomini significava "esser stati a Bwamba", dove i Bakonzo celebravano il rito insieme ai Baamba, che a loro volta, si dice, l'avevano appreso dai pigmei Batwa, stanziati al di là del Rwenzori nella foresta congolese dell'Ituri ${ }^{24}$.

41 All'inizio di questa ricerca il ricordo del tempo in cui le stagioni dell'olhusumba si aprivano periodicamente era ancora molto vivo fra coloro che avevano più di cinquant'anni. Quando, in base a certi segni, veniva indetta una stagione, la voce si diffondeva rapidamente e i villaggi organizzavano i gruppi di iniziandi da mandare in foresta per essere circoncisi e per apprendere quel che serve a un uomo adulto di sapere. Lo stato di liminalità durava un paio di mesi ed era accompagnato non solo dagli iniziatori presenti nel campo (samba: v. oltre), ma dall'intero villaggio, che doveva osservare una serie di prescrizioni rituali. Pur senza comportare cambiamenti troppo drastici - rifiutati, in generale, dalla società konzo, che preferisce metabolizzare le mutazioni con processi lenti - il rito "faceva" uomini adulti, separandoli nettamente dalle donne e dai bambini.

L'ultima stagione iniziatica che ha coinvolto l'intero bukonzo si è svolta all'inizio degli anni '60. Nel decennio seguente, nell'intento di islamizzare l'intero paese, Idi Amin ordinò la cessazione di tutti i riti tradizionali. I Bakonzo non avevano particolari questioni politiche contro il dittatore, che risiedeva nella lontana Kampala e a cui dovevano la creazione del distretto di Kasese. L'olhusumba venne dunque sospeso, dapprima senza particolari problemi dato il suo andamento temporale erratico; dopo 
una quindicina d'anni, tuttavia, cominciò a porsi la questione dell'accesso dei maschi allo status di adulto.

43 A questo problema le due zone del bukonzo risposero in maniera differente. A nord, attorno a Bundibugyo, un'ulteriore stagione iniziatica completa fu organizzata all'inizio degli anni '70. In seguito, pratiche iniziatiche su scala ridotta si mantennero fino all'inizio di questo secolo: gruppi di adulti, parenti degli iniziandi, riunivano i bambini, li circoncidevano e poi li tenevano nello stato liminale per un periodo di un paio di settimane, durante il quale svolgevano, in forma accelerata, la maggior parte della pedagogia iniziatica. Per contro nel distretto di Kasese l'esigenza iniziatica si trasformò nella questione di essere o meno circoncisi - poco importa se l'intervento era fatto da gruppi ambulanti di circoncisori oppure in ospedale. Questa trasformazione, tuttavia, si è riflessa sul senso stesso della cosa: oggi essere circoncisi non significa più "essere stati fatti uomini" (Remotti 2011), ma serve a distinguere i bambini "puliti" da quelli "sporchi" e i Bakonzo da quasi tutti i gruppi circostanti.

In ciò s'innestano il diffondersi del monoteismo (in particolare di quello cristiano) e la scolarizzazione. «Prima c'erano gli spiriti a guidare la nostra vita», affermò un'anziana intervistata insieme a un gruppo di donne del villaggio di Nyakalengigyu nell'estate del 2005. «E ora?» «Ora siamo cristiani, abbiamo Gesù», tradusse subito l'informatrice; la trascrizione dell'intervista, però, riportava: «Ora abbiamo l'erisoma». Nel lhukonzo di oggi il termine erisoma (traducibile letteralmente con "leggere e scrivere") indica tanto la scolarità quanto la religiosità monoteista: una polisemia estremamente significativa, che illumina due meccanismi fondamentali della logica antropopoietica moderna.

Istituita nel 1997 dal presidente Museveni, la Universal Primary Education consente a tutti i bambini di frequentare gratuitamente la scuola pubblica (fatte salve le tasse che, di fatto, tutte le scuole richiedono): nel giro di tre anni la percentuale di bambini iscritti è balzata dall'11\% all' $85 \%$ e oggi sfiora il $99 \%$. Fino ad allora, chi decideva di entrare nel sistema di istruzione scolastica si trovava ad abbandonare completamente le modalità tradizionali: spesso doveva trasferirsi in città e l'accesso stesso a scuola richiedeva, fra l'altro, di essere vestiti ${ }^{25}$. Così, mentre una maggioranza di bambini girava nuda per $\mathrm{i}$ villaggi in attesa dell'iniziazione, una minoranza cominciava a sperimentare il valore antropopoietico della scuola e aveva quindi meno bisogno di un rito di passaggio. Oggi tutti i bambini sono tenuti a frequentare le scuole elementari e, nel discorso sociale, si ritiene che maschi e femmine abbiano le stesse capacità $\mathrm{e}$ debbano studiare il più a lungo possibile. Il transito per il sistema scolare è dunque passaggio fondamentale nella formazione dei Bakonzo solo da una ventina d'anni: la sua capacità di messa in forma, che apre a tutti una serie di nuove possibilità nel campo dei lavori salariati, ancora non sostituisce però, per i maschi, la poiesis operata dal rito, mentre mette le femmine di fronte a una biforcazione inedita che in alcune circostanze, come vedremo, può rivelarsi critica.

Allo stesso modo la conversione al cristianesimo ha avuto, per molti adulti konzo, un forte valor antropopoietico, segnando una cesura netta nella loro vita. A lungo il battesimo ha rappresentato un passaggio in grado di modificare l'essenza stessa delle persone, conferendo loro una nuova forma di umanità e affiliandoli a un'ecumene che li connette - in quanto singoli e non in quanto appartenenti a un gruppo - alla dimensione globale. E poiché una tale trasformazione richiede una certa consapevolezza, esso generalmente si svolgeva, come un tempo l'olhusumba, quando il battezzato aveva raggiunto un'età sufficiente per avere coscienza del rito. La nettezza 
del passaggio ha cominciato a farsi assai meno evidente con le due generazioni seguenti, quelle dei figli e dei nipoti dei primi convertiti, per i quali l'appartenenza a una chiesa universale non è più una scelta. Nondimeno, per molti Bakonzo oggi viventi la transizione, e talora l'opposizione, fra un "prima" e un "dopo" è ancora molto viva, così come le questioni che sono nate dall'attrito fra i due sistemi e le mediazioni necessarie a risolverle.

\section{L'omusitu, gli spiriti e i confini del parco}

L'olhusumba è anche detto "andare in foresta" (Crupi 2008; Facci 2009) e l'omusitu, parola che traduce tanto il nostro concetto di "foresta" quanto quello di "montagna", è fra i Bakonzo un attaccamento fondamentale (Alnaes 1998). A differenza dei Banande, che si narrano come "abbattitori di alberi" e mantengono nei confronti della foresta un sentimento di timore, i Bakonzo - che abitano al suo interno, modificandola - si descrivono come "figli della foresta": è l'omusitu a fornire nutrimento, combustibile, legname e bambù per la costruzione delle abitazioni, erbe e piante medicinali, nonché $\mathrm{i}$ materiali per gli strumenti dell'orchestra endara. Ma soprattutto, la foresta è luogo privilegiato di relazione con gli spiriti che vi dimorano.

Affine al più vasto kubandwa diffuso nell'area dei Grandi Laghi e caratterizzato dalla possessione spiritica (Chrétien 1985; Pennacini 1998; Behrend, Luig 1999), l'eribandwa ${ }^{26}$ konzo è popolato di spiriti che abitano luoghi inusuali (Pennacini 2000). Quando vi si riferiscono, i Bakonzo parlano di omulimu (pl. abalimu), termine che traducono con spirits, ma preferiscono riferirvisi con espressioni come other people o those who own us. I più potenti abitano le vette delle montagne: Nyamuhanga, il creatore, col quale gli umani non hanno rapporti ${ }^{27}$; Kithasamba, "spirito bianco" pericoloso da avvistare ${ }^{28} \mathrm{e}$ leader del pantheon, padre spiritico degli albini, padrone della guerra e del Rwenzori, che risiede sui ghiacciai più alti insieme alla sposa/sorella Nyabingi; Nyabibuya, protettrice dei bambini insieme a Mulemberi e madre spiritica dei gemelli, che devono esserle dedicati; poi Kalisya, protettore della caccia, Ndioka, che abita le acque e può annegare le persone, e altri spiriti che proteggono diverse attività. Oltre a questi, il pantheon konzo è popolato dagli spiriti degli elementi della natura, degli animali selvatici e degli antenati, che spesso entrano in contatto con i vivi attraverso azioni di disturbo volte a ricordare agli umani i loro doveri rituali.

All'eribandwa, cui si ricorre quando è necessario individuare la causa di un disordine o ricostituire una forma di ordine, tutti prendono parte. Le cerimonie si svolgono nella capanna rituale dell'embandwa, il medium (uomo o donna) che funge da sacerdote del rituale di possessione. Il rapporto con gli spiriti è mediato dalla musica: lo strumento principale è l'endara, uno xilofono a barre mobili suonato insieme ai tre tamburi engoma (Crupi 2008). Per via della sua evidente funzione di connessione con gli spiriti, l'endara che un tempo era presente ovunque le persone si riunissero: nelle celebrazioni e nei rituali, ma anche di sera, dopo il pasto, nei cortili - è andato scomparendo e oggi è limitato quasi solo alle funzioni comunitarie officiate dagli embandwa ${ }^{29}$.

Sostanzialmente benevolo ma non scevro di pericoli che aumentano gradualmente con l'avvicinarsi alla neve e ai ghiacciai, l'omusitu dev'essere rispettato e una serie di norme ne regolamento l'accesso. Nei boschi a ridosso delle abitazioni i bambini possono giocare e pascolare le capre; le donne in età fertile possono percorrere gli stretti sentieri che portano agli appezzamenti coltivati, ma di solito non superano il confine 
segnato dalla crescita spontanea di taluni alberi ad alto fusto. Da qui fino ai boschi di bambù si avventurano gli uomini e le donne anziane, oppure quelle dotate di specifiche protezioni, per raccogliere funghi, erbe medicinali, miele, e gli uomini anche per cacciare piccola selvaggina. Oltre il bambù, nel territorio proibito alle donne, vanno solo i cacciatori di professione fino al confine della neve: il loro rientro nei villaggi è regolato da rituali di purificazione e di astensione dai rapporti sessuali per evitare di portare in seno alle famiglie gli spiriti degli animali cacciati o delle entità non umane che potrebbero avere incontrato, o contrariato, in quota.

Nel 1940 gran parte dell'omusitu e delle pianure a sud e a sud-est della catena montuosa furono ricompresi nei confini del Rwenzori Mountain National Park e del Queen Elisabeth National Park, parchi dall'estensione enorme che hanno inglobato quasi tutto il territorio al di sopra dei 1600-1800 m. La popolazione che lo abitava e lo frequentava fu spinta verso valle: Ibanda fu edificata negli anni ' 80 per rispondere alle esigenze dei gruppi sfollati dai crinali. Per diversi decenni i confini rimasero porosi e, seppure in misura minore, i Bakonzo continuarono a cacciare e raccogliere materie prime nell' omusitu, nonché a trovarvi rifugio in occasione di lotte e insurrezioni. Le cose precipitarono definitivamente nel 1992, quando l'Uganda Wildlife Authority decise di rinnovare le strutture e la regolamentazione dei parchi, delimitandone i confini con esogeni e infestanti eucalipti ad alto fusto e ingaggiando guardiaparco armati che, in varie circostanze, hanno sparato su bracconieri e semplici raccoglitori trovati in zone vietate.

Funzionali alla conservazione naturalistica, alla creazione di un'area-cuscinetto fra Uganda e RDC e, soprattutto, al turismo, i parchi hanno costretto i Bakonzo fuori dalle aree montane, confinandoli a un territorio molto più ridotto. In Uganda è una storia che si ripete: come nei casi più celebri degli Ik e dei Batwa (Turnbull 1972, Lewis 2000), e pur con effetti un po' meno disastrosi, anche fra i Bakonzo i nuovi limiti logistici hanno avuto un impatto notevole sulla vita pratica e spiritica, rendendo pressoché impossibili la caccia, la raccolta dei prodotti della foresta e lo svolgimento delle pratiche tradizionali. All'interno dei parchi sono rimaste intrappolate le sommità dei crinali, dove si trovano le tombe degli antenati, ormai poco riconoscibili perché ricoperte di foresta. Da qui iniziavano i rituali annuali di purificazione che garantivano l'abbondanza dei raccolti, l'igiene dei rapporti con le entità spiritiche, la ridistribuzione delle risorse e il consolidamento della struttura politica territoriale. Il rapporto con gli spiriti, che un tempo avveniva in modo privilegiato nell'omusitu, oggi può avvenire solo più nella capanna rituale degli embandwa.

L'esistenza del parco e del turismo che esso attrae spiega quella del Rwenzori Mountaineering Services, l'ente interamente gestito dai Bakonzo che, fino al 2015, era il solo sul territorio a organizzare le scalate alle vette e a formare guide alpine e portatori. Le opportunità salariali connesse al turismo non hanno però ancora sovrascritto la specifica relazione konzo all'omusitu. Nonostante i bianchi lo facciano, secondo i Bakonzo nessun essere umano dovrebbe metter piede sui ghiacciai dove dimorano le divinità: le guide alpine sono i passeurs di un confine fra mondi molto più sottile e pericoloso di quello naturalistico visibile ai turisti. 


\section{Spiriti contra Spirito}

54 La frontiera del parco, con la sua ridefinizione burocratica della vita spiritica konzo, ha sviluppato una dinamica autonoma: facilitando la penetrazione del cristianesimo nelle aree a ridosso del confine (Zavaroni 2008b), ha creato due dominî contrapposti: quello degli spiriti del Rwenzori all'interno del parco e quello dello Spirito Santo al suo esterno.

55 A cascata, ciò ha generato una ridefinizione dell'eribandwa e degli embandwa. La parte più modernizzata della società konzo tende a non menzionare medium, rituali di possessione e spiriti in genere se non in termini dispregiativi. Poiché il vocabolo embandwa implica il rapporto con gli spiriti, esso viene spesso sostituito dalla parola omuthahwa, "guaritore", la stessa che si usa anche per i medici dei dispensari e che, pur non escludendole, non allude alle pratiche stregonesche implicite nel termine embandwa. Chi pratica, o è sospettato di praticare, prevalentemente la stregoneria è definito omuloi, "stregone": nessuno affermerebbe apertamente di essersi rivolto a un omuloi, mentre dire di avere fatto ricorso a un omuthahwa significa per lo più aver cercato aiuto in una circostanza critica.

In un contesto in cui tutti conoscono tecniche per proteggersi dalle intenzioni avverse e per colpire i nemici, tuttavia, ciò che distingue coloro che rientrano nel continuum omuthahwa/embandwa/omuloi dalle persone comuni, oltre al fatto di essere più potenti, è la loro capacità di entrare in comunicazione con il mondo spiritico, ottenendone informazioni sulle "cose nascoste". Come altrove nel continente (de Rosny 1981, 2006; Comaroff, Comaroff 1993; Geschiere 1995; Coppo 2007; Bellagamba 2008), anche qui la distinzione tra operatori del bene e operatori del male è funzionale soprattutto alla visione occidentale. Fin dalla primissima espansione coloniale la posizione del cristianesimo è stata di guerra aperta nei confronti di pratiche automaticamente ascritte alla dimensione del male. Tale chiusura è fra le cause della curiosa concomitanza fra diffusione della modernità e diffusione della stregoneria "maligna" (Taussig 2017; Comaroff, Comaroff 1993; Geschiere 1995; Shaw 1997), che l'etnografia Bakonzo conferma: mentre nella tradizionale Bundibugyo c'è una maggior presenza di embandwa, cui ci si rivolge per risistemare l'ordine delle cose e ottenere cura e protezione, nella moderna Kasese ci sono più abaloi, cui ci si rivolge per ottenere denaro e successo o per danneggiare il prossimo, in un'evidente polarizzazione delle pratiche medianiche sul fronte del male.

57 I due dominî - quello degli spiriti della montagna, catturati entro i confini del parco, e quello dello Spirito Santo - coesistono l'uno accanto all'altro in modo non pacificato: ciò che vale da una parte non vale dall'altra, le protezioni che da questo lato riparano dall'altro espongono agli attacchi. Gli informatori raccontano di scorribande notturne degli "spiriti bianchi della montagna" a danno delle popolazioni cristianizzate, così come di indebite penetrazioni dello Spirito Santo nell'omusitu per mezzo di guide alpine e portatori, che incuranti del pericolo, sfidano l'ambiente montano pregando e utilizzando il linguaggio comune, quello dei centri abitati, in luoghi in cui sarebbe invece necessario ricorrere a uno speciale vocabolario rituale.

58 Ancorché poco parlabili, gli spiriti sono però ancora ben presenti a livello consapevole, note le piste per negoziare la relazione con loro ed estremamente attiva la logica antropologica a cui soggiacciono. Detto altrimenti, gli spiriti konzo ancora non sono fantasmi ma attaccamenti noti e riconosciuti, intenzionalità attive le cui richieste 
possono essere diverse da quelle che vengono dalla logica moderna. Un contrasto che può arrivare a essere anche molto acuto, ma che, come vedremo, non è necessariamente destinato a essere pacificato tramite un aut aut.

\section{Lo spazio della crisi}

\section{Muhindo John: l'articolazione necessaria}

Poco dopo i vent'anni Muhindo John ebbe un periodo di crisi che tutti, nella valle di Ibanda, ricordano: girovagava nudo, non si lavava, aveva i capelli incolti e insultava la gente per strada. Matto conclamato, all'epoca trascorse anche un periodo, che i più ricordano come decisamente lungo, presso l'unico ospedale psichiatrico nazionale, quello di Buthabika a Kampala. Quando lo incontriamo per la prima volta, Muhindo è un uomo di circa quarant'anni, sorridente, disponibile, di professione meccanico, sposato e padre di quattro figli; ed evidentemente guarito ${ }^{30}$.

Solo in apparenza esito di pura destrutturazione, anche la follia ha un suo metodo. Fra i Bakonzo è possibile che diverse entità (spiriti della montagna e della natura, antenati ecc.), per loro autonoma volontà oppure sotto la guida di stregoni o altri malintenzionati, aggrediscano una persona causandole una malattia fisica, un disordine della fertilità oppure una forma di follia e finanche la morte. Coloro che sono affetti da erisire ("follia") presentano soprattutto disturbi del comportamento sociale. I matti rifiutano le regole sociali e i canoni dell'adeguatezza: si comportano male, sono testardi, parlano in modo disordinato (in questo caso si definiscono omuthya muthya, "coloro che blaterano"), non hanno cura del corpo, non si tagliano i capelli, vestono in modo improprio oppure circolano nudi, mangiano disordinatamente nutrendosi anche di scarti o di terra. Si tratta della forma più diffusa di follia, detta erisire eryabbulikiro, "follia comune". Vi sono poi coloro che si ritraggono dalla vita sociale, non parlano e restano in disparte: queste persone sono affette da erykamumu, generalmente conseguenza di stregoneria. Muhindo è stato un "folle comune": nel cortile di un ostello di Ibanda - un luogo tranquillo e al riparo da estranei, dov'è possibile affrontare temi difficili - racconta la sua storia.

61 La sua malattia comincia il giorno in cui, recatosi a una riunione del Rwenzori Mountaineering Services per la quale era necessario essere in possesso dell'invito, si accorge all'ingresso di avere con sé solo la busta vuota. Dapprima viene lasciato fuori, poi ammesso. La mattina dopo si sveglia infreddolito, dice a sua madre di non sentirsi bene ed esce per andare in paese a comprare dei medicinali ma, arrivato sulla strada principale, vede il sole farsi sempre più vasto e smette di sapere cosa gli sta succedendo. Comincia allora a compiere azioni incomprensibili, che poi non ricorda: come la volta che, incontrando per strada un militare, se ne prende gioco finché quello estrae una pistola e minaccia di sparargli. In quel momento, dice, ha ripreso conoscenza, come se qualcosa gli fosse caduto di dosso, e ha pregato il soldato di lasciarlo andare.

L'erisire ha due eziologie principali: può derivare da una causa meccanica o fisiologica (in questo caso, la testa non funziona come dovrebbe per via di una malattia quale la malaria cerebrale, oppure a seguito convulsioni o traumi fisici ripetuti, o ancora a seguito del consumo di stupefacenti); oppure - ed è il caso più comune - è provocata dall'intromissione di volontà esterne al malato (spiriti, attacchi stregoneschi, 
maledizioni, chiamate all'eribandwa), che possono avere varie cause, fra cui l'attribuzione al clan sbagliato o il mancato adempimento dei doveri rituali ${ }^{31}$. Si tratta di condizioni in cui la testa del malato non è in alcun modo danneggiata e, se viene attuata la corretta strategia per risolvere lo squilibrio, può riprendere a operare normalmente, senza lasciare alcuna conseguenza né funzionale né sociale: poiché i folli non sono responsabili dei loro comportamenti, quando guariscono non vengono redarguiti. Bisogna fare in fretta, però: se la follia rimane troppo a lungo senza essere curata, allora rovina la testa e diventa inguaribile ${ }^{32}$.

Le vittime di attacchi spiritici sono tra i più comuni pazienti degli embandwa. Il percorso è impegnativo: prevede una lunga degenza a casa del guaritore, con o senza la presenza di un familiare, e la creazione di nuove alleanze. Il rapporto tra l'embandwa e il paziente - così come quelli fra il medium "maestro" e il medium "candidato", fra gli iniziandi e i loro circoncisori, fra la partoriente e la levatrice - si descrive con lo stesso termine reciproco, sambà: ciascuna parte di queste relazioni è sambà dell'altra e nel corso della vita, in occasione di passaggi e funzioni importanti, gli uni saranno invitati a prendere parte alle cerimonie degli altri.

Due mesi dopo l'esordio, i genitori di Muhindo lo portano da un guaritore, dove resta per un mese. Di quel periodo ricorda la madre che gli portava il cibo mentre lui aveva mani e piedi legati: il percorso terapeutico consiste infatti, tra l'altro, in una battaglia tra il guaritore e le entità che vorrebbero impossessarsi del folle e che, contrarie alla sua permanenza dall'embandwa, lo spingono a scappare ${ }^{33}$. Il guaritore lo cura per mezzo di erbe e, tramite possessione medianica, ottiene indicazioni sulla terapia da attuare. La cura tuttavia non ha successo e i familiari decidono di portarlo in ospedale, pur sapendo il problema è connesso agli spiriti: poco prima che il giovane si ammalasse, infatti, suo padre aveva avuto problemi del tutto simili. Nipote di una nota embandwa, l'uomo sarebbe dovuto diventare embandwa a sua volta ma, per via dell'appartenenza religiosa, aveva abbandonato le pratiche rituali senza preoccuparsi di placare gli spiriti. Curato efficacemente da un guaritore tradizionale, una settimana più tardi gli spiriti che avevano tormentavano il padre erano passati al figlio.

All'arrivo all'ospedale di Kilembe, i medici suggeriscono ai genitori di Muhindo di occuparsi in primo luogo di risolvere le questioni familiari e di riportare il giovane in ospedale solo dopo avere sistemato le pratiche tradizionali: «La prima volta che $\mathrm{mi}$ portarono là [i dottori] mi esaminarono, ma la malattia non fu trovata; allora dissero: "riportatelo a casa, il disturbo potrebbe arrivare da cose culturali ${ }^{34}$, prima di tutto lavorate quelle"».

Così è fatto. Fra questa prima, breve ospedalizzazione e quella seguente di otto mesi a Buthabika, Muhindo viene ritrovato nel fiume più di una volta. Ma se è uscito vivo dall'ospedale, dice, è perché prima di riportarcelo i familiari hanno concluso le pratiche tradizionali: il padre ha accettato il mandato come embandwa, ha costruito una capanna sacrificale e ha cominciato a curare pazienti.

67 A Buthabika la cura consiste in pillole, iniezioni e saltuari colloqui. Quando rientra a casa, tutto gli appare nuovo e sconosciuto: la gente va a trovarlo in lacrime e lui si sente come un resuscitato. Ha perso tutto quel che aveva: per abituarsi alla nuova situazione decide di restare per un po' chiuso in casa. Piano piano, la sua vita si risistema.

È comune sul Rwenzori, come altrove, che l'esperienza della follia lasci a chi guarisce conoscenze relative alla cura. Liberato il folle dall'erisire, e mentre lo rieduca alla vita sociale, un buon embandwa insegna al paziente e alla sua famiglia come gestire le 
richieste degli spiriti per evitare ricadute. Se in questa fase l'ex folle manifesta capacità nuove, un buon sambà si occuperà anche di formarlo perché apprenda a utilizzare correttamente le sue nuove conoscenze.

Muhindo riassume la sua guarigione come un susseguirsi corretto di approcci diversi: prima le pratiche tradizionali hanno sgombrato il campo dai rischi spiritici, poi l'ospedale psichiatrico ha rinnovato la sua mente, da ultimo un lungo periodo di riflessione tra le mura domestiche gli ha permesso di riabituarsi alla vita e infine riprendere a lavorare, trovare moglie e avere figli. Col tempo ha scoperto di possedere la capacità e le conoscenze necessarie a curare alcune malattie, tra cui la gotta, le micosi della pelle, gli aborti spontanei e i morsi dei serpenti ai bambini ancora nel ventre della madre. Poiché le informazioni relative alla malattia e alla cura gli arrivano in sogno, senza richiedere la possessione, Muhindo non la considera una forma di eribandwa e non ha quindi avuto bisogno di essere iniziato come guaritore.

Il problema dell'eredità degli spiriti, tuttavia, si è ripresentato quando la morte del padre ha lasciato "scoperta" la posizione dell'embandwa iniziato: all'epoca dell'intervista, due familiari di Muhindo stanno vivendo situazioni di sofferenza e malattia. Gli spiriti chiedono la loro parte: di poter accedere alla vita umana in modo ordinato. Qualche giorno dopo i suoi fratelli, pur riluttanti a parlare di questioni spiritiche, riportano che in effetti una nipote sta patendo pene simili a quelle subite da Muhindo: perché cessino, prima o poi qualcun altro dovrà accettare gli spiriti e diventare embandwa.

\section{Mary N.: affermarsi o fermarsi}

71 All'epoca della prima intervista Mary ha 23 anni e sta terminando un corso per segretarie nella cittadina di Kasese ${ }^{35}$. Fino a un certo punto della sua vita è stata una brava scolara, promettente: la UPE è in vigore da poco più di un decennio e lei appartiene alle prime generazioni cui la scuola si è presentata come nuovo modo per tutti di messa in forma degli umani. Una messa in forma che s'innesta, tuttavia, sopra una rete di legami, vincoli e doveri assai differente da quella ipotizzata dalla pedagogia euro-americana. Anche nel suo caso, come in quello di Muhindo, l'eziologia è familiare.

Il padre di Mary, Paul M.B., è guida alpina di grande esperienza e nipote, per ascendenza maschile, di un embandwa. Suo padre (il nonno di Mary), tuttavia, aveva scelto di tenersi a distanza dalle pratiche tradizionali, di non accettare la chiamata a embandwa ereditata dal padre e di battezzare i figli. Per tutta la vita aveva sostenuto che sarebbe stato meglio per tutti se lui fosse morto da cristiano. Per questo motivo, quando in età avanzata fu colpito da una malattia, i figli convocarono un catechista: questi gli diede una benedizione a seguito della quale l'uomo si ristabilì; in seguito partecipò al catechismo, ricevette il battesimo e infine morì. Da cristiano.

73 A dodici anni Viviane, la madre di Mary, è stata la prima della sua famiglia a essere battezzata. Il padre non l'aveva mandata a scuola perché voleva che seguisse i lavori domestici ma, accortosi che la bambina si era fatta triste, le aveva permesso di seguire il catechismo alla diocesi di Kasese. A quindici anni Viviane sposa Paul con un matrimonio "pulito" (ovvero cattolico), ma nei primi tempi la vita familiare è piuttosto tradizionale. A ridosso del matrimonio partorisce una prima figlia prematura, che non sopravvive, e ci vogliono poi quattro anni di gravidanze divorate dai vermi e le cure di un embandwa perché nasca la seconda figlia. In occasione di questa gravidanza, però, gli 
spiriti cominciano ad assalirla, bloccandola a letto e minacciandola. Quando, contro il suo parere, Paul prende un'altra moglie, la situazione peggiora ulteriormente: fra le due mogli, così come col resto della famiglia, nascono conflitti; i campi non rendono; la guerra tiene lontani i turisti e Paul è spesso impegnato in rischiosi spostamenti in montagna per contrabbandare tabacco, finendo poi per sperare i soldi in alcol e donne.

Dalla seconda moglie Paul ha diversi figli e tutti e tre i coniugi (il marito insieme alle due mogli) vengono iniziati in quanto genitori di gemelli. Come altrove in Africa (Turner 1972; Oruene 1985; Remotti 1993, 1994; Savary, Gros 1995; Renne, Bastian 2001), anche fra i Bakonzo le nascite speciali - parti gemellari o podalici, bambini albini introducono disordine e perplessità in un sistema che si vuole, altrimenti, altamente ordinato e civilizzato e che prevede che da ogni parto nasca un solo bambino. La madre di gemelli viene trattata alla stregua di una capra, qualcuno che ha rotto la barriera fra umani e animali e occorre intervenire sia per placare la capacità dei gemelli di "bruciare", che per togliere i genitori dalla situazione di impurità, iniziandoli come Isebahasa e Nyabahasa (padre/madre di gemelli). È anche un'occasione per acquisire prestigio: i genitori iniziati di gemelli sono reputati più saggi, e quindi liberi di trasgredire alcune norme sociali, e i padri, che durante l'iniziazione ricevono la zucca rituale ekisya, vengono chiamati a praticare rituali di purificazione nelle circostanze contrarie all'ordine corretto della vita della comunità ${ }^{36}$. Paul però, pur iniziato, ha rifiutato di possedere la zucca.

A lungo Paul e Viviane si sono rivolti all'eribandwa per trovare soluzione ai loro problemi. Ma non ha funzionato e le sorti della famiglia parevano disperate. Infine, una decina d'anni prima, una sorta di conversione: Viviane decide di rivolgersi alla chiesa per la cura dei suoi mali; Paul caccia la seconda moglie e, seguendo la prima, si riavvicina al cristianesimo, interrompendo tutte le pratiche tradizionali e arrivando, nel giro di qualche anno, a diventare catechista. È stato allora che gli spiriti hanno cominciato a disturbare Mary.

In quanto gemella, Mary ha una relazione speciale con Nyabibuya, lo spirito che protegge i bambini; e gli spiriti sono presenti fin da subito nella storia del suo strano scacco scolastico. Fra i sette e i tredici anni vive a Kizinga con un'amica della madre che, non avendo figlie femmine a casa, si è offerta di pagarle gli studi. Questa "maternità affidataria" è una pratica diffusa e accettata da tutti, più comune per le femmine che per i maschi, e spesso vantaggiosa per i giovani. I disturbi di Mary cominciano in prima superiore, quando ancora si trova presso la famiglia affidataria, con l'arrivo, nella stessa scuola, di una cugina di lato paterno. Figlia di un fratello minore di Paul, insieme alla madre e a tutti i fratelli la cugina è in rotta con la famiglia del padre. Le due giovani entrano in conflitto: Mary è presa da spiriti che "le vogliono male" e non riesce quasi più ad andare a scuola. Dopo due settimane si trasferisce a Kasese, alla rinomata Bright Academy dove già studia la sua gemella (anch'essa disturbata dagli spiriti). All'ingresso in terza superiore, "l'altra madre" le paga le tasse scolastiche perché frequenti, nei pressi di Kampala, la scuola di cui suo figlio è direttore. Qui tutto procede per il meglio finché un fratello della cugina di cui sopra arriva nella stessa scuola, latore di quei medesimi spiriti che rendono la vita di Mary impossibile. Lei sa di chi si tratta, perché li sogna di notte; ma non ha chiaro perché ce l'abbiano con lei, né se siano gli stessi spiriti che disturbano la sua gemella. Ricominciano i sintomi, questa volta più forti: dolori di testa ricorrenti, dismenorrea, malessere fisico, difficoltà a concentrarsi, temporanei stati di incoscienza. Gli spiriti 
sono gelosi, non vogliono che finisca gli studi, le suggeriscono di buttare i libri nel water.

Lo schema è ricorrente. La UPE è strutturata in sette anni di scuola primaria, seguiti da quattro anni di scuola secondaria, che si concludono con un esame nazionale detto Ordinary Level Examination. Altri due anni di scuola danno accesso all'Advanced Level Examination che prelude agli studi universitari. Capita spesso che le studentesse più brillanti si blocchino in quarta superiore, subito prima degli " $O$ " levels: malesseri, spiriti cattivi, catatonia. Nonostante le campagne per la scolarità femminile e le buone intenzioni del discorso sociale, c'è in gioco qualcosa che non è facile da definire. Cosa succede alle donne che "hanno studiato"?

Avere frequentato alcuni anni di scuola superiore è ininfluente: è ritenuto un buon percorso, ma ancora non significa "avere studiato". A sancire un livello di scolarità elevato, distinto dalla media dei giovani konzo, è l'ingresso nella parte conclusiva delle superiori, il superamento degli "O" levels e la frequenza degli ultimi due anni. Il superamento degli "A" levels fa di un(a) giovane una persona istruita, moderna, che cercherà un impiego stipendiato e forse l'accesso all'università. Nonostante la retorica scolastica inciti tutti a proseguire gli studi, nella realtà sociale molti uomini ci hanno riferito di preferire il matrimonio con donne alfabetizzate, ma prive di un orizzonte professionale extra-domestico. In più, le donne istruite costano: nella lunga transazione fra famiglie che precede il matrimonio, il prezzo della sposa (che compensa il padre degli sforzi economici sostenuti per formare la giovane donna) aumenta a seconda del suo grado di istruzione e $\mathrm{i}$ conteggi possono essere anche molto dettagliati ${ }^{37}$. Una situazione difficile anche per i giovani uomini istruiti, per i quali non sono disponibili i lavori ai quali sono stati preparati e che, quindi, non possono contare su alcuna risorsa supplementare per pagare al suocero un prezzo più alto del normale. Con la conclusione della quarta superiore si aprono quindi per le ragazze due diversi orizzonti: il matrimonio; oppure la possibilità di diventare infermiere, insegnanti, segretarie, suore o addirittura medici o avvocatesse, secondo quanto la scuola le ha sempre spronate a immaginare, ciò che tuttavia le rende mogli meno appetibili anche da parte degli uomini colti, quelli che loro stesse desidererebbero come compagni di vita.

Mary finisce comunque gli studi, seppure non tanto bene quanto ci sarebbe attesi, torna a Kasese e, da quel momento, le cose un po' si sistemano. La situazione familiare, tuttavia, resta delicata e il problema di Mary, pur relegato sullo sfondo, non è effettivamente risolto. Suo fratello Arnold è certo che tutta la famiglia sia coinvolta in un unico disordine spiritico, ereditato dalle generazioni precedenti: Viviane, infatti, è ancora disturbata da crisi spiritiche, che cerca di abbreviare attraverso la preghiera. Entrambi i genitori confermano la possessione delle loro figlie da parte di spiriti, ma non li mettono in relazione con quelli della madre, né hanno mai voluto identificarne l'origine: sono stufi di occuparsene o, forse, fanno finta di niente. Pregano con costanza e dedizione, evitano di commettere peccati e pensano che anche le figlie trarrebbero giovamento da un avvicinamento al cristianesimo ma, come afferma la madre, solo $\mathrm{i}$ diretti interessati possono scegliere la fede - il che significa, in definitiva, rinunciare a proteggere la figlia secondo i modi previsti dalla logica tradizionale.

80 Al contrario dei suoi genitori, Mary parla apertamente dell'opposizione degli spiriti alla sua affermazione personale. Nella relazione col fidanzato ha scelto di nascondere la sua parte debole e pericolosa, quell'eredità familiare di possessione e follia che la 
renderebbe poco appetibile come moglie; e di accettarlo come marito solo se le permetterà di finire il corso da segretaria che sta frequentando.

81 La giovane si è trovata a combattere la sua battaglia piuttosto sola, sebbene il sostegno economico della "seconda madre" le abbia consentito un'autonomia di cui molte altre giovani nella sua condizione non godono. La personale strategia che ha messo in atto si basa sulla scommessa di una convivenza fra attaccamenti e modelli: il fatto stesso che Mary riconosca l'esistenza di diverse tensioni in contrasto le permette di gestire attivamente e autonomamente le diverse risorse. È quindi riuscita a elaborare un modo per partecipare agli esami e a concludere il primo ciclo della scuola superiore nonostante gli spiriti continuassero a disturbarla. Utilizza la preghiera come "rimedio sintomatico" per tenere a bada le volontà avverse, ma senza negare la potenza e le intenzioni di queste ultime. E anche rispetto al fidanzamento, in autonomia dalla famiglia la giovane ha messo in atto una strategia che le assicuri un futuro consentendole sia di essere moglie, sia di avere una certa indipendenza professionale.

\section{Adele K.: in carico a nessuno}

82 La forma di follia che ha colpito Adele non è acuta come quella di Muhindo, ma di lunga durata: disturbata in modo intermittente da molti anni, Adele è sposata, ha figli e generalmente si comporta in modo socialmente accettabile ${ }^{38}$. Periodicamente arrivano le crisi, che approdano ogni volta a una mancata soluzione.

Il nome che porta racconta già una parte della storia. Il sistema nominale konzo prevede oggi che al nome tradizionale, che indica la posizione di nascita rispetto al gruppo dei fratelli uterini, si aggiungano un nome inglese (che spesso è anche il nome di battesimo) e un numero variabile di nomi circostanziali (Zavaroni, Consigliere 2018). Adele è dunque la primogenita della seconda moglie del sig. P. Mumbere e il suo nome circostanziale significa, in lhukonzo, "sofferenza". Di lei si racconta infatti che sia nata dopo una gravidanza durata due anni: la madre era stata colpita da un obuthambi, una maledizione spiritica che si manifesta, in questo caso, con serpenti nel ventre che ostacolano le gravidanze, rendendole intermittenti. Mumbere aveva cercato di farla curare dapprima in ospedale, poi presso un guaritore, ma in occasione della seconda gravidanza la donna aveva deciso di divorziare portandosi via il bambino, che era poi nato zoppo. In seguito il nuovo marito ha fatto curare la donna, liberandola dall' obuthambi, e la coppia ha potuto avere quattro figli sani. Adele è stata cresciuta dalla prima moglie di Mumbere come una figlia propria: i genitori raccontano di averle nascosto chi fosse sua madre per non disturbarne l'infanzia.

In seconda elementare, all'incirca verso i dieci anni, un giorno Adele viene trovata in lacrime, febbricitante, che grida. Il padre, recentemente battezzato nella Church of Uganda, la porta in ospedale e qui i medici diagnosticano una forma di malaria cerebrale che curano per due settimane, senza però guarirla. Seguendo il consiglio di altre persone, che ritenevano si trattasse di erisire, Mumbere fa ricorso all'eribandwa e, per l'occasione, consulta due diversi embandwa: il primo, tramite il rituale di possessione, indica nell'aggressione da parte di spiriti dell'acqua ${ }^{39}$ la causa della follia, mentre il secondo si occupa dell'effettiva terapia, purificando la bambina che, il giorno stesso, guarisce. Per completare l'azione terapeutica questo secondo embandwa deve dare alla bambina un nuovo nome protettivo, ma muore prima di compiere il rito, lasciando a metà il percorso di cura. 
85 Adele resta in salute abbastanza a lungo da arrivare, qualche anno dopo, a sposarsi. Consapevole di maritare una figlia malata, come prezzo della sposa Mumbere chiede al futuro marito solo tre capre (a fronte delle tradizionali dodici) e una piccola somma di denaro. Alla nascita del primogenito c'è la prima ricaduta, che inaugura una lunga alternanza di crisi e tentativi di cura. Il marito pretende dal padre la restituzione del prezzo, che non ottiene dacché la donna gli ha comunque dato un figlio; allora la riporta a casa del padre, perché sia questi a sostenere le spese per le cure. Curata, ha col marito un secondo figlio; nuovamente si ammala; e nuovamente è restituita al padre. A questo punto un altro uomo fugge con Adele senza rimborsare le capre al primo marito e incorrendo quindi in una multa che, mai pagata, lo rende marito illegittimo. Del resto, come spiega Mumbere, egli non avrebbe potuto richiedere un nuovo prezzo della sposa al secondo marito, essendo un padre tenuto a "mangiare le capre" una sola volta, e un suo tentativo di fare collaborare tra loro i due uomini sarebbe naufragato. Con il secondo marito Adele ha tre figli, ma anche in questo caso senza essere pienamente assunta come moglie. Le crisi si ripetono. È l'epoca della guerra, Adele va e viene da famiglie che non sono mai completamente sue: «E allora tutte quelle cose disturbano la testa ogni volta che penso ai miei figli e alle loro sofferenze; a volte li lascio in mezzo al conflitto e non c'è pace», dice.

Le strategie di cura cambiano nel tempo: l'ospedalizzazione, l'eribandwa, la preghiera, di nuovo la medicina convenzionale. Qualcosa, da qualche parte, sembra rimasto in sospeso: l'ospedale non cura o è troppo caro, la preghiera non è sufficiente, l'eribandwa sembra evitato. Cambiano anche le spiegazioni per il mancato ricorso alle terapie tradizionali. In un primo tempo Mumbere e la moglie dicono di aver interrotto tutte le pratiche spiritiche nel 1986, quando sono stati battezzati in seno alla Church of Uganda: «I cristiani dicono che si deve credere in un solo Dio che può curare qualsiasi male $\mathrm{e}$ solo a questi si deve chiedere aiuto». Da solo, Mumbere spiega invece che la malattia di Adele dipendeva dell'obuthambi di sua madre, la malattia connessa agli spiriti dell'acqua: la bambina sarebbe guarita completamente se l'embandwa che l'aveva curata avesse fatto a tempo a darle un nome speciale - o se, alla morte di questi, il padre ne avesse consultato un altro. Per sua parte, Adele propone una terza motivazione: le entità che la disturbano sono spiriti di famiglia, connessi con le pratiche claniche; quando comincia a stare male vede in sogno l'ekyaghanda, la capanna rituale che il padre dovrebbe costruire per lei.

87 La storia di Mumbere sembra confermare l'ipotesi eziologica di Adele. Nel passato, quando il bisnonno paterno di Adele era capo-clan, la famiglia manteneva un' ekyaghanda, alla cui manutenzione Mumbere avrebbe dovuto provvedere alla morte del nonno. Mumbere, però, non ha ascoltato la chiamata degli spiriti del clan, né compiuto sacrifici in onore dello spirito cacciatore della pianura a cui la famiglia è legata. Anche in questo caso, le motivazioni addotte sono diverse. La più immediata è che oggi c'è il cristianesimo e tutti hanno rinunciato alle pratiche tradizionali. Una seconda spiegazione, connessa alla prima ma di diverso tenore, è che, per avere una capanna sacrificale, è necessario avere una prima moglie che possa fungere da Nyabalimu (madre degli spiriti) e sappia quindi preparare l'obundu per il banchetto da offrire agli spiriti e oggi è impossibile trovare una moglie adatta a questo compito. Un terzo motivo, emerso alla fine di uno scambio acceso tra padre e figlia, sta nel fatto che Mumbere, primogenito del primo figlio del defunto capo-clan, ha zii paterni minori (vale a dire fratelli minori del padre) ancora in vita: per costruire una capanna sacrificale è 
necessario l'accordo di tutto il clan, ma se egli convocasse una riunione per discutere se assumere su di sé le incombenze rituali, che pure a suo dire gli spetterebbero, potrebbe essere tacciato di volersi imporre sugli altri - faccenda doppiamente delicata, dal momento che alcuni membri del suo clan ricoprono cariche politiche e territoriali di rilievo e certo non desidererebbero riconoscergli un ruolo di prestigio.

Quanto a Adele, sembra non sia mai stata presa pienamente in carico da nessuno: la madre la lascia al padre e non tornerà mai a conoscerla (sarà la figlia, ormai sposata, ad andare a cercarla); il guaritore che avrebbe potuto attribuirle il nome protettivo muore e non viene sostituito; il padre non assume gli obblighi clanici; i mariti non concludono i matrimoni, mantenendosi liberi di restituire la donna in caso di malattia. La posizione è sempre ambivalente: mentre tutti riconoscono che occuparsi delle questioni familiari permetterebbe a Adele di guarire, tutti, inclusa lei stessa, affermano che ormai resta soltanto la preghiera.

\section{Strategie del molteplice}

\section{Cautele}

Nessun confronto contenutistico può essere fatto fra quanto va accadendo fra $\mathrm{i}$ Bakonzo del Rwenzori (con i loro attaccamenti specifici, il ricordo ancora vivo dei nessi e delle conseguenze stabiliti dallo loro logica antropopoietica, la diplomazia spesso faticosa fra appartenenze e obblighi) e quanto si vede accadere fra i migranti (con l'opacità e spesso l'inconoscibilità dei loro quadri di riferimento, il traumatismo del viaggio, le condizioni totalizzanti del trattenimento, le negoziazioni con la società locale). Perfino nel caso in cui a sbarcare sulle nostre coste fosse un mukonzo, si dovrebbe comunque ipotizzare che il tragitto migratorio e le condizioni dell'accoglienza e della permanenza nella società d'arrivo abbiano agito e agiscano come dispositivi di parziale riplasmazione antropopoietica. Non è dunque al livello dei contenuti espliciti, o esplicitabili, che bisogna cercare le somiglianze, ma più sotto, dove i processi antropopoietici inscrivono precocemente l'esito di una scelta fondamentale: quella fra monismo e molteplicità, fra possibilità di convivenza fra mondi e volontà di unificazione. Spostando qui l'attenzione, ci sono forse alcune indicazioni che si possono trarre dall'osservazione congiunta di questi due campi o, se si preferisce, di queste due storie postcoloniali.

Spiriti-fantasma, confinati sulle vette del Rwenzori dai limiti di un parco, che col linguaggio dei sogni, della possessione, della follia chiedono di essere onorati; spiriti dai molti nomi, "altre persone" di cui nessuno parla a cuor leggero con un musungu per timore di un giudizio o dell'ennesimo richiamo a ciò a cui un buon credente è tenuto. $\mathrm{Ma}$ anche ombre di violenza coloniale e geopolitica, sospetti di stregoneria o di rapimento, dubbi angoscianti sulla parte indicibile dei racconti e l'ossessione della verità in quanto insieme di fatti positivamente accertabili, l'insostenibilità di ciò che chiamiamo progresso così come del venir meno del suo mito, l'adesione consapevole $\mathrm{e}$ inconsapevole alle parole d'ordine della cosmovisione egemone: tutto questo si mobilita nell'incontro forzato della migrazione, nelle strategie per resistere all'annullamento culturale o allo spaesamento. Eppure, è solo ammettendo l'esistenza e l'azione dei fantasmi che li (e ci) attraversano che si fa possibile immaginare modi per uscire dall'impasse. 


\section{Peripezie dell'adultità}

91

92 dal rituale dell'olhusumba, mentre le femmine seguivano una progressione più graduale, gravidanza ${ }^{40}$, che le avrebbe confermate come donne e mogli. In ogni caso, era assai chiara la demarcazione fra chi è adulto e chi non lo è. Oggi il superamento con buoni voti degli " $O$ " levels apre effettive prospettive nella modernità, ma non è sufficiente per conferire agli individui in una forma adulta pienamente riconosciuta. La scuola forma ma non dà forma, non è garanzia di adultità raggiunta e, nel caso degli uomini, non rappresenta un valido sostituto delle iniziazioni rituali. La cessazione dell'olhusumba ha liberato i maschi dalla pressione a partecipare a un rituale potenzialmente pericoloso e diminuito il rischio immediato che devono affrontare; questo, tuttavia, non garantisce loro un aumento di possibilità e di potenza e rende la loro evoluzione più lenta e meno certa - incertezza che si fa acuta in un panorama di guerra, scontri col governo, assenza di lavoro salariato e difficoltà, se non impossibilità, a procurarsi mogli adeguate. L'accesso degli uomini all'età adulta non è sancito dalla circoncisione, trasformatasi in un mero segno di appartenenza al gruppo; non viene raggiunto col percorso scolastico; e non sta neppure nella genitorialità che, spesso, arriva prima del matrimonio. L'ultimo passaggio tuttora disponibile che conserva un valore antropopoietico certo sembra essere il matrimonio, con l'assunzione di responsabilità che esso comporta.

Ancora più significativo è il caso delle donne. Il modello sociale tradizionale, che pure sanciva una separazione piuttosto netta dei compiti tra generi, prevedeva per tutti uomini e donne - un contesto esistenziale sostanzialmente familiare; nel caso delle donne si trattava della famiglia del marito, presso cui si trasferivano in occasione del matrimonio o, in caso di alleanze di lunga durata fra famiglie, anche prima. In quel quadro, tuttavia, era riconosciuta anche alle donne (fin da bambine o, per chi compiva scelte specifiche, a partire dalla prima adolescenza) la possibilità di sottrarsi alle limitazioni della vita domestica attraverso l'acquisizione di conoscenze e competenze specifiche, che accrescevano sensibilmente il loro prestigio sociale: potevano lavorare come levatrici, erbaliste, embandwa, artigiane del vasellame o commercianti, senza che ciò le rendesse spose o madri inadeguate. Si trattava in ogni caso, e come per gli uomini, di attività di relazione con il resto della collettività, che consolidavano gli attaccamenti comunitari pur consentendo un'affermazione extra-domestica. Oggi alle giovani è offerta, attraverso la scuola, la possibilità moderna di affermarsi fin da subito su una base individuale, che tuttavia le mette in contrasto con un "loro", gli uomini, con cui è più difficile confrontarsi.

94 Infine, sebbene la costruzione individuale veicolata dalla scolarità agisca in modo del tutto simile su maschi e femmine, gli esiti sono alquanto diversi: per i primi esiste uno spazio sociale capace di accogliere, entro certi limiti e con un certo sospetto, questo tipo di affermazione; per le altre, al contrario, non esiste un reale campo d'azione se non a fronte di notevoli rinunce, dacché la loro costruzione extrascolastica continua a situarle in seno alle relazioni familiari e coniugali e alla maternità. Succede così che a 
una certa età i maschi si blocchino e le femmine si ammalino. Le crisi che spesso emergono verso la fine del percorso scolastico, come nel caso di Mary, non derivano dalla difficoltà di pensarsi contemporaneamente professioniste e mogli, ma dal fatto che, in quel contesto, scarseggiano uomini che accettino mogli professioniste: un po' perché una donna meno istruita "costa meno", un altro po' perché, per gli uomini che svolgono attività professionali extra-domestiche, la moglie funge anche da attaccamento alla collettività, ancorando marito e figli al contesto sociale.

In questo quadro, l'adesione al cristianesimo rappresenta una sorta di punto mediano: mentre la scolarità e il lavoro salariato proiettano il soggetto in un orizzonte di cui non si percepiscono i confini, i sistemi religiosi lo accolgono in un contesto locale coeso e strutturato, benché "modernamente" esteso a livello globale. Per le donne, inoltre, essa offre il vantaggio di potersi attivare autonomamente, senza bisogno che, come nei rituali tradizionali, a mobilitarsi sia un intero gruppo.

Se ora ci spostiamo nel contesto migratorio, emergono subito due zone esperienziali in cui il tema dell'accesso all'età adulta è cruciale: quello dell'inserimento nei percorsi scolastici o formativi e quello dell'autonomia delle donne. Capita spesso di vedere giovani uomini, sopravvissuti a quella vera e propria iniziazione al nero che è il viaggio verso le nostre coste, condannati dall'anagrafe a frequentare una specifica classe elementare o media - e quindi a ritrovarsi gettati anni indietro rispetto alla maturità raggiunta "sul campo" e al mandato con cui sono partiti. Dal punto di vista normativo, in effetti, non c'è null'altro che si possa offrire loro: l'obbligo scolare è, da noi, l'unica pista antropopoietica disponibile. La storia stessa di questi ex-bambini, tuttavia, nonché gli esiti del loro inserimento fra coetanei cresciuti in Italia, richiederebbe l'invenzione di qualcosa di nuovo, piste che non li rigettino in una minorità coatta che, a volte, arriva a uccidere ${ }^{41}$.

Allo stesso modo, nel contesto migratorio chi non intenda aderire senza riserve alla proposta antropopoietica dell'Italia contemporanea, ha l'esigenza che almeno uno dei due coniugi (quello meno impegnato nel salariato, e quindi solitamente la donna) resti saldo nella logica di provenienza, per poter ancorare l'intero gruppo familiare anche a un'altra appartenenza, vissuta come piena di senso, dotata di passato e di futuro e origine di attaccamenti irrinunciabili (la lingua madre, la strutturazione pulsionale, le relazioni di ascendenza ecc.). Ben oltre la canonica opposizione fra integrazione e separatismo, è la dinamica stessa dei processi culturali e biografici a richiedere il massimo rispetto per gli attaccamenti primi: così come la piena competenza in almeno una lingua madre permette l'accesso ad altre lingue, anche l'accesso alla modernità qualora venga scelto - non può che partire da una competenza culturale altra, piena $\mathrm{e}$ strutturante. Si tratta dunque di mantenere due modelli, entrambi rilevanti nel presente biografico dei migranti, in una compresenza non troppo faticosa.

\section{Onorare gli attaccamenti}

Come molte altre cosmovisioni rese subalterne dalla modernità, la logica konzo prevede che la strutturazione fondamentale dei soggetti passi attraverso legami stretti e vincolanti con la comunità e che il mondo sia popolato di entità non-umane intenzionate (antenati, spiriti ecc.). La logica moderna, per contro, privilegia l'autonomia dei soggetti e la libertà come allentamento dei legami, limitando la possibilità di essere persona ai soli umani e ipotizzando la non-esistenza, oppure 
l'intrinseca malevolenza, di qualsiasi entità non-umana intenzionata. Nell'analisi di questa differenza antropopoietica si può fare riferimento ai concetti elaborati nell'antropologia della persona (CNRS 1973; Comaroff, Comaroff 2001) e alla terminologia introdotta da Strathern (1988) e pensare quindi all'individuo come soggetto "essenzializzato", conchiuso in sé stesso, e al dividuo come soggetto costitutivamente attraversato dalle relazioni. Illuminante e feconda (Lambeck, Strathern 1998; Bird-David 1999), questa proposta manifesta oggi un paio di criticità: da un lato, anziché farne i poli di un campo continuo, tende a opporre i due termini e quindi ad agire come principio rigido di classificazione; dall'altro, come nota Sahlins (2013: 36), «si coglie una tensione irrisolta fra l'enfasi riservata alle persone dividuali e le relazioni, poste in certa misura sullo sfondo, che le costituiscono». Nella prospettiva adottata possiamo ipotizzare che i processi antropopoietici implementino diverse logiche di individuazione, che intersecano in punti diversi il continuum fra dividuo e individuo (Capello 2016) e producono quindi diversi modi di attaccamento: prevalentemente orientato alla tessitura di una rete relazionale quello che i Bakonzo riconoscono come proprio, prevalentemente orientato alla relazione di sé a sé quello euro-americano.

99 Le donne e gli uomini Bakonzo hanno trovato molti modi personali per scendere a patti con le diverse istanze che queste due logiche mobilitano, o per ignorarle, in un contesto dove lo stigma ontologico, epistemologico ed etico su tutto ciò che è "non moderno" (ciò che, direbbero loro, è cultural) continua a farsi sentire con una carica notevole di violenza simbolica. Ma appunto: modi personali, vie di fuga individualizzate, spesso trovate per prova-ed-errore dopo anni di sofferenza, di follia, di tensione, di incertezza. Il fatto è, però, che gli spiriti konzo, come quelli di tutto il kubandwa, non ragionano in termini di individuo ma in termini di famiglia; non si curano del singolo in quanto tale, ma della congiuntura specifica che lo qualifica in un clan o in un lignaggio; e, se colpiscono qualcuno, è per mandare un messaggio all'intero gruppo dei suoi.

100 Fra i Bakonzo di oggi il quadro modernizzante offerto dalla chiesa e dalla scolarità lascia scoperto un fatto cruciale: se il cristianesimo può limitare gli umani rispetto agli spiriti, esso non può limitare gli spiriti rispetto agli umani. Come in molti altri contesti nei quali gli invisibili esercitano una continua influenza sulla vita degli umani, anche nel contesto konzo la modernità non rappresenta una realtà che scalzi, in modo repentino e definitivo, quella precedente, ma un orizzonte di relazioni e vincoli che stratifica su quello locale. Il senso del nuovo quadro è negoziato a partire dalle categorie di quello già attivo.

101 Forse è proprio questo che la giovane Mary ha chiaro, a differenza di molte persone delle generazioni precedenti: se la sua vita è determinata da una molteplicità di attaccamenti (la scolarità, il cristianesimo, gli spiriti konzo), ciascuno corrispondente a un diverso progetto, non è possibile limitarsi ad affrontare solo uno di questi progetti, rispondere a una sola delle richieste negando le altre. Ciascun attaccamento richiede un suo tempo, una sua cura, e poiché non tutti gli ambiti le consentono di agire autonomamente, cerca di adoperarsi a partire da ciò che le è possibile.

102 Come evidenziano tutte e tre le storie raccontate, la soluzione dell'erisire non sta solo nella cura di chi ne è colpito, ma nella risoluzione delle questioni che percorrono e inquinano il livello familiare da parte di chi ha i titoli per farlo. È dunque spesso qualcun altro a dover compiere le azioni indispensabili - accettare la chiamata a embandwa, costruire una capanna rituale, farsi carico di una posizione di potere - 
perché "le altre persone", ottenendo ciò che è loro dovuto, si plachino: «La gente che va in chiesa abbandona la cultura. Mettiamo che hai un padre catechista: è lui che sa le cose della cultura, però non le segue. Allora, anche se non ne sai niente, gli spiriti vengono da te $\mathrm{C}^{42}$ ».

Qui, negli interstizi fra logiche, s'innestano dinamiche complicate. Molte delle storie raccolte evidenziano una certa disparità di genere surrettiziamente introdotta dalla modernità. Quando gli squilibri interni a un gruppo familiare si manifestano attraverso la sofferenza di un membro debole (una donna giovane, un bambino), gli uomini della famiglia - i soli titolati a risolvere la questione - possono permettersi di non agire, evitando riposizionamenti familiari troppo radicali, limitandosi a pregare e tenendosi quanto più possibile lontani dall'affrontare le cause che motivano la richiesta spiritica. Nel caso di Adele, il nonno embandwa muore poco prima della conversione di Mumbere al cristianesimo: inevitabile interrogarsi su quanto il cristianesimo, con l'anatema che fa cadere sulle pratiche tradizionali, non sia anche un buon motivo per evitare di affrontare la scomoda questione familiare ${ }^{43}$. Non a caso, mentre Muhindo - grazie anche a un'articolazione terapeutica di estrema intelligenza - guarisce, le crisi di Adele e Mary sono invece cronicizzate.

Quando gli operatori lamentano che, nelle famiglie migranti, i mariti non consentono alle mogli di integrarsi nel paese ospitante - di "emanciparsi", nel nostro vocabolario leggono questo comportamento come un antico retaggio patriarcale o come doppio standard morale. In ogni caso, attribuiscono senz'altro a entrambi i coniugi lo stesso statuto di "autonomia a-relazionale" che vale per noi. Così, il solo intervento possibile a favore delle donne che vivono situazioni familiari difficili prevede il rinforzo dell'autonomia individuale, indipendentemente dal background esistenziale e dalla rete degli attaccamenti vitali: comunità protette madre-bambino, inserimento lavorativo, assunzione di una posizione salariata. Autonomia individuale che, tuttavia, spesso non si può neanche cominciare a costruire senza prima mettere a posto le questioni che pertengono all'individuazione relazionale - questioni, però, che è spesso qualcun altro (e non il soggetto stesso) a essere titolato a trattare. Un esempio: quando una madre migrante e un figlio minore vengono separati dal padre senza tentare la pista della mediazione familiare, lungi dal proteggere univocamente il minore, il provvedimento rischia di metterlo nella posizione di chi, in assenza della figura paterna, spesso titolata a portarvelo, non potrà accedere alla posizione adulta secondo i canoni vigenti nella cultura familiare, esacerbando le difficoltà relative al processo di filiazione e, come ricaduta, a quello di affiliazione (Ahovi, Moro 2010).

\section{Il «dio geloso» e la crisi}

La compresenza di due diversi insiemi di attaccamenti non sembrerebbe, di per sé, portare un maggior rischio di crisi e per gli individui in evoluzione non si dà un'intrinseca impossibilità a incarnare più progetti diversi. Semmai, a far problema sono il rifiuto, l'incapacità o l'impossibilità di onorare uno dei due insiemi, laddove la molteplicità sia attivamente rifiutata, o combattuta, da una delle logiche compresenti laddove, cioè, uno o più set di attaccamenti si vogliano come esclusivi. Per metafora: non è impossibile a una singola persona possedere più passaporti; la questione si pone quando uno dei paesi eroganti impone un'appartenenza esclusiva. 
In un gran numero di casi osservati la crisi si apre, per la persona direttamente interessata o per un suo familiare, perché "non sono state fatte le cose che bisognava fare". Per loro stessa natura molteplici, gli spiriti konzo non sembrano risentirsi per il nuovo attaccamento alla chiesa o al paradigma moderno; ma non danno pace ai mortali quando coloro che sono tenuti a farlo cessano di alimentare il rapporto di scambio tra umani, spiriti e territorio. Ciò equivale a dire che, dal vertice osservativo della pratica dell'eribandwa, è possibile essere al contempo buoni cristiani e "buoni Bakonzo", rispettosi tanto del Dio biblico quanto degli spiriti konzo; il fatto è che, come molti guaritori confermano, la chiesa non lo consente.

Il campo lontano ha evidenziato molte volte che le "cose" della tradizione sono poco pubblicamente parlabili. In ciò bisogna leggere un elemento di pudore e protezione nei confronti dei rappresentanti della logica moderna, ma anche la polarizzazione della tradizione sul versante del negativo: non solo ciò che è vecchio e superato, ma anche ciò che è intrinsecamente ambiguo, pericoloso, incerto. Se dunque il discorso pubblico moderno squalifica le pratiche tradizionali come superstizioni, la chiesa vi mette sopra un vero e proprio veto in quanto maligne e la scienza medica in quanto dannose. Una situazione del tutto simile s'incontra anche nel contesto d'arrivo dei percorsi migratori che, decisamente meno plurale di quanto non ci piaccia ammettere, non riconosce spazi possibili per le espressioni multiple di un singolo individuo.

Sebbene la situazione sia in lento miglioramento, attualmente tanto nell'accoglienza quanto nella vita ordinaria la logica di prima appartenenza dei migranti è ben poco parlabile, se non nella più superficiale e commerciale delle maniere (cibi e vestiti "etnici", musica "tribale" ecc.). Non solo: se viene parlata in modo incauto, rischia di scatenare conseguenze gravi, che spaziano dallo stigma al ricovero in psichiatria. L'idea del salvataggio materiale, ma soprattutto culturale, dei bisognosi che sbarcano sulle nostre coste è ancora molto attiva fra gli operatori, inclusi quelli dal posizionamento politico più attento: è una delle tracce potentemente impresse sull'inconscio collettivo da quattro secoli di colonialismo, mai veramente lavorati - come se il postcolonialismo fosse una questione che riguarda solo i colonizzati.

Ora, proprio questo rigetto pubblico di un insieme di attaccamenti attivi è uno degli elementi che più concorrono alla cronicizzazione delle crisi, tanto nel luogo d'origine quanto nel contesto migratorio. Dove queste derivino dal mancato rispetto dei doveri nei confronti degli attaccamenti locali, il veto sulle possibili piste di risoluzione fa sì che le crisi stentino a trovare una via di scioglimento.

110 La non esclusività dei quadri di riferimento è cruciale per i percorsi di cura. La complementarità tra medicina occidentale e eribandwa è alla base della guarigione di Muhindo, circostanza fortunata in cui nessuno degli attori in gioco ha imposto l'esclusività del proprio quadro di riferimento. A considerazioni del tutto analoghe sono giunte anche le sperimentazioni più avanzate nell'articolazione dei sistemi terapeutici (Coppo, Keita 1990; Chan 2008; OMS 2013). In ambito etnopsichiatrico si è perfino ipotizzato che la compresenza di attaccamenti multipli sia garanzia di una pluralità di traiettorie possibili, sia nelle normali circostanze della vita, dove essa consente adattamenti più elastici, sia in caso di crisi, quando la molteplicità permette di ragionare a partire da più piste possibili di lavorazione e risoluzione (Grandsard 2005; Sironi 2017). Qui la parte urgente del lavoro da fare non riguarda affatto la popolazione migrante, ma quella ospitante, a cui servono strumenti concettuali ed emotivi per 
superare lo spaesamento culturale e accettare di abitare un mondo intrinsecamente molteplice.

\section{Per concludere, o per ricominciare}

111 L'osservazione congiunta di quanto sta avvenendo nei due "campi postcoloniali" considerati permette così di trarre qualche conclusione. La prima è questa: nella complessa molteplicità degli attaccamenti e delle logiche culturali, spesso i soggetti riescono a costruirsi su binari plurali che non sono di per sé intrinsecamente problematici. La problematicità di questa costruzione, talvolta tanto grave da dare origine a crisi profonde, deriva semmai dalle pretese totalizzanti di una di queste logiche.

112 A fronte di costruzioni multiple - oggi motivate dalla migrazione, dal rapporto tra orizzonti di senso che si incontrano e si sovrappongono, ma anche dal nostro stesso bisogno di uscire dalle gabbie del paradigma moderno - si pone la questione di non considerare come fondante un solo set di attaccamenti (magari proprio quello tradizionale, in un goffo tentativo di rimediare al colonialismo delle anime), ma di prendere in considerazione la capacità degli umani di essere costruiti simultaneamente secondo traiettorie diverse, di essere connessi con il mondo attraverso attaccamenti plurali (ciò che include inevitabilmente epistemologie e ontologie alternative), provando a costruire luoghi in cui la pluralità sia manifestabile e valorizzata, senza la pretesa di ridurla a forme monodimensionali.

Aujourd'hui, il nous faut adapter et complexifier notre approche à l'égard des problématiques cliniques contemporaines car, plus que jamais auparavant, les sujets sont à la fois reliés et déliés, inscrit dans une dimension locale (culturelle, politique, professionnelle) et globale (mondialité des échanges, internet, circulation planétaire des hommes et des idées...). La multiplicité de nos appartenances (politique, culturelle, sociale, professionnelle, religieuse ou laïque) devient alors une force, même si elle peut parfois, et de manière provisoire, produire des symptômes (Sironi 2017: 30).

La seconda indicazione è questa: quando la crisi colpisce un soggetto costruito da due (o più) logiche di attaccamento, privilegiarne una negando l'altra (o le altre) fa diminuire le probabilità di risoluzione. Nella presa in carico di queste crisi si hanno maggiori probabilità di successo quando nessuno dei terapeuti coinvolti impone un quadro di riferimento unico sul disordine che occorre sanare, e quando tutti permettono che diversi quadri di riferimento si confrontino senza escludersi (Bouznah, Lewertowski 2017). Ciò non vuol dire rinunciare alla funzione antropopoietica del percorso terapeutico - rinunciare, cioè, a condurre socialmente i nuovi individui verso una forma matura, capace, presente di umanità o a ri-metterli in forma a seguito di una crisi di presenza (Kakar 1995); vuol dire, piuttosto, mettersi in cerca di processi capaci di strutturare senza intrappolare; di percorsi di cura che, nel ridare una forma, non prendano in ostaggio i pazienti.

114 Operazioni tecniche di questo tipo, comuni in ambito etnopsichiatrico, hanno sollecitato molte riflessioni - talora anche violentemente critiche - sullo statuto ontologico degli "invisibili" degli altri e sullo spaesamento che essi causano ai nostri operatori, messi di fronte a possessioni, attacchi stregoneschi, riti per placare Mami Wata, dolori senza causa organica apparente ecc. Comprensibili, dunque, le richieste di rassicurazione sulla superiorità ontologica delle nostre categorie e dei nostri invisibili 
(ivi inclusi, e anzi in primis, quelli terapeutici - l'inconscio, ad esempio), che descriverebbero la realtà in modo universale, misurabile e ovunque verificabile, a scapito delle categorie esotiche degli altri, concetti magico-infantili, buoni al più per il loro portato simbolico: il "credere" di chi non ha ancora a disposizione gli strumenti per "sapere". Ma si tratta di una manovra difensiva, una sorta di auto-protezione psicologica che rischia di fare danni: a coloro che vorremmo accogliere e alla possibilità, nostra e loro, di divenire altro.

\section{BIBLIOGRAFIA}

Agamben G., 2003 Stato di eccezione, Bollati Boringhieri, Torino.

Ahovi J., Moro M.R., 2010 «Rites de passage et adolescence», in Adolescence, 2010/4 (74): 861-871.

Alnaes K., 1967 «Nyamingi's Song: an Analysis of a Konzo Circumcision Song», in Africa: Journal of the International African Institute, 37(4): 453-465.

Alnaes K., 1998 «The Snow as the Centre of the Konzo Universe», in H. Osmaston, J. Tukahirwa, C. Basalirwa, J. Nyakaana (eds), The Rwenzori Mountains National Park, Uganda, Dept. of Geography, Makerere University, Fountain Publishers, Kampala: 288-299.

Arce A., Long N., 2000 Anthropology, Development, and Modernities, Routledge, London.

Bäärnhielm S., Laban K., Scouler-Ocak M., Rousseau C., Kirmeyer L.J., 2017 «Mental health for refugees, asylum seekers and displaced persons: A call for a humanitarian agenda», in Transcultural Psychiatry, 54 (5-6): 565-574.

Balinandi Kambale Fr. R., 2002 Lhukonzo English Dictionary (edizione privata).

Baer H.A., Singer M., Susser I., 1997 Medical anthropology and the world system. A critical perspective, Bergin \& Garvey, Westport (USA) \& London.

Baluku Bakahinga Mbalibulha S., 2008 «Ethicity, the state and the Dynamics of Ethnopolitical Conflict in Western Uganda», paper presentato al Dipartimento di Scienze Antropologiche, Archeologiche e Storico-Territoriali dell'Università di Torino, inedito.

Baubet T., Moro M.R., 2009 «Effets de la migration et de l'exil», in T. Baubet, M.R. Moro (eds), Psychopathologie transculturelle, Elsevier Masson, Issy-les-Moulineaux: 49-57.

Behrend H., Luig U. (eds), 1999 Spirit possession, Fountain Publishers, Kampala.

Bellagamba A., 2008 L'Africa e la stregoneria, Laterza, Bari-Roma.

Beneduce R., 1998 Frontiere dell'identità e della memoria. Etnopsichiatria e migrazioni in un mondo creolo, Franco Angeli, Milano.

Beneduce R., 2016 «Traumatic pasts and the historical imagination: Symptoms of loss, postcolonial suffering, and counter-memories among African migrants», in Transcultural Psychiatry, 53 (3): 261-285.

Bergner G., 2009 «Black Children, White Preference: Brown v. Board, the Doll Tests, and the Politics of Self-Esteem», in American Quarterly, 61 (2): 299-332. 
Bird-David N., 1999 «"Animism” Revisited: Personhood, Environment and Relational Epistemolgy», in Current Anthropology, XL, Supplement: 67-91.

Blommaert J., 2009 «Language, Asylum, and the National Order», in Current Anthropology, 50 (4): 415-441.

Bouznah S., Lewertowski C., 2017 Quando gli spiriti incontrano i medici (ed. or. 2013), Colibrì, Paderno Dugnano.

Buffa M., 2015 Trattenuti e trattamenti. Esistenze e spazi fra penale e non penale, tesi di dottorato, Scuola di Dottorato in Scienze Giuridiche, Università degli Studi di Milano.

Capello C., 2016 Antropologia della persona. Un'esplorazione, Franco Angeli, Milano.

Chan M., 2008 «Alocucion ante el Congreso de la OMS sobre medicina tradicional», Beijing, novembre 2008, WHO/OMS.

Chrétien J.P., 1985 «L'empire des Bacwezi. La construction d'un imaginaire géopolitique», in Annales ESC, 40(6): 1335-1371.

CNRS§, 1973 La notion de personne en Afrique noire. Colloque International du CNRS, Paris, L'Harmattan.

Comaroff J., Comaroff J.L., 1992 Ethnography and the historical imagination, Westview Press, Boulder. Comaroff J., Comaroff J.L., 1993 Modernity and Its Malcontents, University of Chicago Press, Chicago. Comaroff J., Comaroff J.L., 2001 «Sul concetto di persona. Una prospettiva dall'Africa», in S. Consigliere (a cura di), Mondi multipli vol. 2, Kaiak, Napoli: 129-150.

Comaroff J., Comaroff J.L., 2009 Ethnicity Inc., University of Chicago Press, Chicago.

Consigliere S., 2014 Antropo-logiche. Mondi e modi dell'umano, Colibrì, Milano.

Cooke P., Doornbos M., 1982 «Rwenzururu Protest Song», in Africa, 52 (1): 37-60.

Coppo P., Keita A. (eds), 1990 Médecine traditionnelle. Acteurs, itinéraires thérapeutiques, Ed. "E", Trieste.

Coppo P., 2003 Tra psiche e culture. Elementi di etnopsichiatria, Bollati Boringhieri, Torino.

Coppo P., 2007 Negoziare con il male, Bollati Boringhieri, Torino.

Coppo P., 2013 Le ragioni degli altri, Cortina, Milano.

Crupi V., 2008 «The Role and Functions of the Endara Xylophone among the Bakonzo People», in C. Pennacini, H. Wittenberg (eds), Rwenzori, Histories and cultures of an African Mountain, Fountain Publishers, Kampala: 253-284.

Da Col G., Graeber D., 2011 «Foreword. The return of ethnographic theory», in HAU - Journal of Ethnographic theory, 1 (1): vi-xxxvii.

Danowski D., Viveiros de Castro E., 2014 Esiste un mondo a venire? Saggio sulle paure della fine, Nottetempo, Roma 2017.

de Martino E., 1977 La fine del mondo. Contributo all'analisi delle apocalissi culturali, Einaudi, Torino. De Pury S., 2005 Comment on dit dans ta langue ? Pratiques ethnopsychiatriques, Les Empêcheurs de penser en rond, Paris.

De Rosny E., 1981 Les yeux de ma chèvre, Plon, Paris.

De Rosny E., 2006 Justice et sorcellerie, Khartala, Paris. 
Delille E., 2016 «On the history of cultural psychiatry: Georges Devereux, Henri Ellenberger, and the psychological treatment of Native Americans in the 1950s», in Transcultural Psychiatry, 53 (3): 392-411.

Descola P., 2005 Par-delà neture et culture, Gallimard, Paris.

Devereux G., 1967 De l'angoisse à la méthode dans les sciences du comportement, Aubier, Paris.

Devereux G., 2007 Saggi di etnopsichiatria generale (ed. or. 1973), Armando Editore, Roma.

Eickelman Dale F., 1985 Knowledge and Power in Morocco, Princeton University Press, Princeton.

Erny P., 2001 Istruzione, educazione familiare e condizione giovanile in Africa, L'Harmattan Italia, Milano.

Facci S., 2009 «Dances across the boundary: Banande and Bakonzo in the twentieth century», in Journal of Eastern African Studies, 3 (2): 350-366.

Falk Moore S., 1994 Anthropology and Africa, University of Virginia Press Charlottesville.

Gardoncini S., 2009 «Crinali. Spazio e potere tra i Bakonzo», in Africa, LXIV (3-4): 508-528.

Gardoncini S., 2010 «Il regno Rwenzururu. I Bakonzo nella modernità», in Africa, LXV (1-4): 330-350.

Geschiere P., 1995 The Modernity of Witchcraft. Politics and the occult in postcolonial Africa, University of Virginia Press, Charlottesville.

Gifford P., 1999 African Christianity: its public Role in Uganda, Fountain Publishers, Kampala.

Ginzburg C., 1979 «Spie. Radici di un paradigma indiziario», in A. Gargani (a cura di), Crisi della ragione, Einaudi, Torino: 57-106.

Good B.J., 1999 Narrare la malattia. Lo sguardo antropologico sul rapporto medico-paziente (ed. or. 1994), Edizioni di Comunità, Torino.

Gordon F.A., 1997 Ghostly matters. Haunting and the sociological imagination, University of Missensota Press, Minneapolis \& London.

Gordon R.G. Jr. (ed.), 2005 Ethnologue: Languages of the World, fifteenth edition, SIL International, Dallas, Texas. Online version: http://www.ethnologue.com/15.

Graeber D., 2015 «Radical alterity is just another way of saying 'reality'. A reply to Eduardo Viveiros de Castro», in HAU Journal of Ethnographic theory, 5 (2): 1-41.

Grandsard C., 2005 Juifs d'un côté. Portraits de descendants de mariages entre juifs et chrétiens, Le Seuil/Empêcheurs de Penser en Rond, Paris.

Heald S., 1999 Manhood and morality: sex, violence and ritual in Gisu society, Routledge, London.

Holbraad M., Pedersen M.A., 2017 The ontological turn. An anthropological exposition, CUP, Cambridge.

Ingold T., Pallson G. (eds), 2013 Biosocial becomings. Integrating social and biological anthropology, CUP, Cambridge.

Jourdan L., 2010 Generazione Kalashnikov. Un antropologo dentro la guerra in Congo, Laterza, RomaBari.

Kakar S., 1995 Sciamani, mistici e dottori (ed. or. 1982), Pratiche Editrice, Parma.

Khamalwa W., 2004 Identity, Power, and Culture: Imbalu Initiation among the Bamasaba in Uganda, Bayreuth African Studies, 68. 
Kleinmann A., 1980 Patients and healers in the context of culture. An exploration of the borderland between anthropology, medicine, and psychiatry, University of California Press, Berkeley.

Kleinmann A., 1995 Writing at the margin. Discourse between anthropology and medicine, University of California Press, Berkeley.

Kohn E., 2013 How forests think. Towards an anthropology beyond the human, University of California Press, Berkeley.

Lambeck M., Strathern A. (eds), 1998 Bodies and persons. Comparative perspectives from Africa and Melanesia, Cambridge University Press, Cambridge.

Latour B., 1994 «Nota su taluni oggetti capelluti», in I Fogli di ORISS, 29-30 (2008): 62-78.

Latour B., 2000a «Factures/fractures. De la notion de réseau à celle d'attachement», in A. Micoud, M. Pironi (ed), Ce qui nous relie, Éditions De l'Aube, La Tour d'Aigues: 189-208.

Latour B., 2000b «Guerra dei mondi - offerte di pace», in S. Consigliere (a cura di), Mondi multipli. Vol. 1. La Grande partizione, Kaiak, Napoli 2014: 165-182.

Lewis J., 2000 The Batwa pygmies of the great lakes region, Minority Rights Group International, London.

Magezi M.W, Nyakango T.E., Aganatia M.K., 2004 The People of the Rwenzori. The Bayira (Bakonzo/ Banande) and Their Culture, Rudiger Koppe Verlag, Koln.

Marcus G.E., Fischer M.M.J., 1999 Anthropology as cultural critique. An experimental moment in the human sciences (ed. or. 1986), University of Chicago Press, Chicago \& London.

Melandri E., 2004 La linea e il circolo. Studio logico-filosofico sull'analogia (ed. or. 1968), Quodlibet, Macerata.

Nathan T., 2003 Non siamo soli al mondo (ed. or. 2001), Bollati Borighieri, Torino.

Nathan T., Stengers I., 1996 Medici e stregoni (ed. or. 1995), Bollati Boringhieri, Torino.

OMS, 2013 Estrategia de la OMS sobre Medicina Tradicional 2014-2023, WHO/OMS, Ginevra.

Oruene T., 1985 «Magical Powers of Twins in the Socio-Religious Beliefs of the Yoruba», in Folklore, 96 (2): 208-216.

Pennacini C., 1998 Kubandwa. La possessione spiritica nell'Africa dei grandi laghi, Il Segnalibro, Torino.

Pennacini C., 2000 «Religion and Spirit Possession in the Great Lakes' Africa», in F. Remotti (a cura di), Ambienti, lingue, culture: Contributi della Missione Etnologica in Africa Equatoriale, Edizioni dell'Orso, Alessandria: 119-150.

Pennacini C. (a cura di), 2006 I popoli della Luna, The People of the Moon, Rwenzori 1906-2006, Edizioni Museo Nazionale della Montagna, Torino.

Pennacini C., H. Wittenberg (eds), 2008 Histories and Cultures of an African Mountain, Fountain Publishers, Kampala.

Rastello L., 2010 La frontiera addosso, Laterza, Roma-Bari.

Remotti F., 1982 «I Banande dello Zaire», in M. Arioti (a cura di), Uomini e Re. Saggi di Etnografia, Laterza, Bari-Roma.

Remotti F., 1993 Etnografia Nande. Vol. I, Società, matrimoni, potere, Il segnalibro, Torino.

Remotti F., 1994 Etnografia Nande. Vol. II, Ecologia, Cultura, Simbolismo, Il segnalibro, Torino.

Remotti F., 2002 Forme di umanità, Mondadori, Milano. 
Remotti F., 2011 Cultura: dalla complessità all'impoverimento, Laterza, Roma-Bari.

Remotti F., 2013 «Dall'identità alle somiglianze: un percorso a ritroso», in Berggasse 19 (9): 133-152.

Renne E.P., Bastian M.L., 2001 «Reviewing Twinship in Africa», in Ethnology, 40 (1): 1-11.

Sahlins M., 2013 What kinship is - and is not, University of Chicago Press, Chicago and London.

Savary C., Gros C., 1995 Des jumeaux et des autres, Musée Ethnographique de Genève, Genève.

Scheper-Hughes N., Lock M.M. (eds), 1987 «The mindful body. A prolegomenon to future work in medical anthropology», in Medical Anthropology Quarterly, 1: 6-41.

Scott J.C., Tehranian J., Mathias J., 2002 «The production of legal identities proper to states: the case of the permanent family surname», in Comparative Studies in Society and History, 44: 4-44.

Shaw R., 1997 «The Production of Witchcraft/Witchcraft as Production: Memory, Modernity, and the Slave Trade in Sierra Leone», in American Ethnologist, 24 (4): 856-876.

Shorter E., 1992 From paralysis to fatigue. A History of Psychosomatic Illness in the Modern Era, Free Press, New York.

Simpson A. (ed.), 2008 Language \& National Identity in Africa, Oxford University Press, Oxford.

Singleton M., 2017 «The Bombardier Marabout of Dakar-An African Shaman? Towards a Nominalist Anthropology», in Anthropos, 112 (2): 499-516.

Sironi F., 2017 Comment devient-on tortionnaire? Psychologie des criminels contre l'humanité, La Découverte, Paris.

Sorgoni B., 2013 «Chiedere asilo. Racconti, traduzioni, trascrizioni», in Antropologia, 15: 131-151.

Stacey T., 2003 Tribe. The Hidden History of the Mountain of the Moon, London, Stacey International.

Stengers I., $2009 \mathrm{Au}$ temps des catastrophes. Résister à la barbarie qui vient, Les Empêcheurs de penser en rond/La Découverte, Paris.

Strathern M., 1988 The Gender of the Gift. Problems with Women and Problems with Society in Melanesia, University of California Press, Berkeley.

Syahuka-Muhindo A., 1991 The Rwenzururu Movement and the Democratic Struggle, Centre for Basic Research Publications, Kampala.

Syahuka-Muhindo A., 2008 «Migrations and social formation in the Rwenzori region», in C. Pennacini, H. Wittenberg (eds), Rwenzori, Histories and cultures of an African Mountain, Fountain Publishers, Kampala: 18-58.

Turnbull C.M., 1972 The Mountain People, New York, Simon \& Schuster.

Taussig M., 2017 Il diavolo e il feticismo della merce. Antropologia dell'alienazione nel capitalismo (ed. or. 1980), DeriveApprodi, Roma.

Taussig M., 1987 Shamanism, Colonialism and the Wild Man: a Study in Terror and Healing, University of Chicago Press, Chicago.

Tsing A.L., 2015 The Mushroom at the End of the World. On the Possibility of Life in Capitalist Ruins, Princeton University Press, Princeton.

Turner V., 1972 Il processo rituale (ed. or. 1969), Morcelliana, Brescia. 
Vallarino Gancia F., 2006 Scenari di cura e di senso: ricerca sui metodi di cura tradizionali attraverso la documentazione fotografica di una terapia tradizionale con un guaritore konzo in Uganda, tesi per il Master di II livello in Etnopsichiatria, Università degli Studi di Genova, a.a. 2005-2006.

Viveiros de Castro E., 2002 «The Relative Native», in HAU Journal of Ethnographic Theory, 3 (3): 473-502.

Viveiros de Castro E., 2009 Métaphysiques cannibals, PUF, Paris.

Zavaroni C., 2008a «Tra Scuola e Tradizione: un caso africano», in Intercultura, 24.

Zavaroni C., 2008b «From the forest to the hospital: Changing circumcision practices among the Bakonzo of the Rwenzori», in C. Pennacini, H. Wittemberg (eds), Histories and Cultures of an African Mountain, Kampala, Fountain Publisher: 200-222.

Zavaroni C., Consigliere S., 2018 «Primo e i suoi fratelli. Il sistema nominale konzo fra relazionalità e individualità», in ANUAC (in corso di stampa).

Zorzetto S., 2016 «Funzione biopolitica delle frontiere nell'accoglienza a richiedenti asilo e rifugiati», in Cambio. Rivista sulle trasformazioni sociali, VI (12): 215-229.

\section{NOTE}

1. Questo articolo è l'esito organico del lavoro congiunto e dividuale delle due autrici. Qualora la macchina burocratica imponesse, una volta di più, il sacrificio di ciò che è intero tramite un'artificiosa disgiunzione dei suoi elementi, la prima sezione «La teoria necessaria» e la quarta «Strategie del molteplice» possono essere attribuite a Stefania Consigliere, la seconda sezione «Spiriti in guerra» e la terza "Lo spazio della crisi» a Cristina Zavaroni. I temi presentati e il taglio della discussione hanno beneficiato, lungo gli anni, della frequentazione di una manciata di luoghi che, in modi diversi, intrecciano antropologia, psicologia e pensiero critico: l'associazione ORISS di Pisa, il Centro Studi Sagara, la sezione di antropologia dell'Università degli Studi di Genova, il Centro Mamre di Torino. A questi spazi, a coloro che li popolano e all'esperienza che vi sedimenta va la nostra più profonda gratitudine. Ringraziamo inoltre la redazione di AAM, nella persona della prof.ssa Gabriella D'Agostino, e i due referees anonimi per la pazienza, l'attenzione critica e la precisione delle osservazioni, che ci hanno costrette a un passo avanti teorico e a tentare "un modo altro" di dire le cose.

2. Nonostante la "refugizzazione" burocraticamente imposta per accedere al permesso di soggiorno, le ragioni economiche ancora motivano la maggioranza delle esperienze migratorie.

3. Uno sguardo d'insieme sull'apparato italiano dell'accoglienza è emerso dai lavori del seminario intitolato «Refugizzare», organizzato dall'associazione ORISS di Lari (PI) e svoltosi nell'aprile 2017.

4. Per un'analisi aggiornata della salute mentale nella popolazione rifugiata o richiedente asilo, v. voll. 46 (4) e 54 (5-6) di Transcultural Psychiatry.

5. In questo senso, troviamo sensato contrastare la logica della modernità, in quanto dinamica egemone e prepotentemente unificante (sia nei fatti che nella cosmovisione soggiacente: Consigliere 2014), alle logiche locali, che la modernità stessa incessantemente produce come subalterne (Latour 1994).

6. È questo, a nostro avviso, il valore dell'ontological turn: nel suo richiamo all'alterità radicale rende evidente l'arroganza di pensare il disincanto come unico viatico della storia (Descola 2005; Viveiros de Castro 2009). 
7. Qui, tra l'altro, si avverte in modo particolarmente acuto la mancanza di un codice deontologico vincolante condiviso dall'intera comunità antropologica (e quindi, ad esempio, di linee guida univoche sul segreto professionale in situazioni cliniche).

8. Senza dimenticare che l'etnografia è necessariamente una pratica analogica: sulla possibilità che logica e analogia costituiscano un campo continuo, anziché un fronte oppositivo, resta imprescindibile Melandri 2004.

9. Per ottenere asilo, documenti e qualche diritto civile sul suolo nazionale, bisogna rientrare in una delle categorie previste dalla normativa (perseguitati politici o religiosi, vittime di discriminazione razziale o sessuale ecc.). Occorre quindi convincere la commissione territoriale del proprio buon diritto producendo una storia credibile (Blommaert 2009; Sorgoni 2013) avventurandosi, quindi, in un genere letterario specifico, ai cui canoni dovranno aderire non solo la relazione scritta, ma anche le risposte alle domande della commissione territoriale: chi dice di provenire da un luogo turbolento dovrà allora conoscerne la geografia minimale così come essa viene insegnata nelle nostre scuole (nomi delle vie, distanze fra insediamenti ecc.); chi afferma di essere perseguitato in quanto minoranza sessuale dovrà rispondere nel modo previsto all'esposizione alla pornografia; chi dice di trovarsi, nel proprio paese, in immediato pericolo personale dovrà fornire prove accertabili adeguate agli ascoltatori (evitando accuratamente di fare riferimento a cose come la stregoneria, che sposterebbero il racconto verso un genere letterario inviso alle "scientifiche" commissioni). E via dicendo.

10. In particolare: educazione tradizionale e impatto della Universal Primary Education; dinamiche di genere in relazione alla modernità; riti d'iniziazione maschili e femminili; nascite e matrimoni; sistemi di cura tradizionali e mediumship embandwa; educazione morale tradizionale e letteratura orale; funzione rituale dell'orchestra endara; nascite di origine spiritica (albini e gemelli); confini del Rwenzori Mountain National Park e suoi rapporti con la comunità spiritica; pratiche rituali connesse con le morti all'interno dei confini del parco; salute mentale.

11. Tre missioni (dicembre 2009/gennaio 2010, luglio/agosto 2010 e giugno/luglio 2017) sono state dedicate a questo tema. Le storie di cui abbiamo una narrazione abbastanza precisa, raccolta da più fonti, sono una quindicina e includono due guaritrici tradizionali; un ex-paziente dell'ospedale psichiatrico nazionale ugandese; un bambino vittima dell'attacco spiritico di un congiunto morto; un giovane che gli spiriti stavano chiamando alla funzione di medium; due uomini e una donna attribuiti a clan "sbagliati"; una donna vittima di attacco cannibalico; una giovane donna che per nascita e clan non appartiene all'etnia konzo ma che gli spiriti della nonna (konzo) stavano chiamando alle pratiche di cura; varie studentesse in stallo; alcuni casi che la comunità definisce di "follia"; e infine due storie di uomini che hanno attraversato lunghe fasi di blocco.

12. Qui larga parte della permanenza è stata ospitata dalla locale parrocchia cattolica; in un paio di missioni, tuttavia, per non subire troppo fortemente l'associazione con una delle tre denominazioni cristiane presenti, è stato preferibile affittare una casa nel villaggio. A partire da Ibanda la ricerca è stata condotta in quasi tutto il Bukonzo (il territorio konzo), accompagnata per il primo anno e mezzo di permanenza dall'informatore più competente, un professore di scuola superiore appartenente alla chiesa avventista, mentre per un'altra parte ha coinvolto l'anglicana Church of Uganda. In questo modo sono state stabilite relazioni con tutte le principali chiese, che si spartiscono equamente la popolazione cristianizzata. Pur in espansione, l'Islam è ancora scarsamente presente, mentre la percentuale di popolazione che pratica esclusivamente il culto spiritico (v. oltre) è stimata intorno all' $8-10 \%$.

13. Dove la permanenza complessiva è stata molto più breve e priva di appoggi logistici istituzionali: tre missioni brevi per un totale di un mese di permanenza.

14. I Bakonzo si trovano anche, ma in parte minore, nel distretto di Kabarole e in quello, di recentissima costituzione, di Bunyangabu. 
15. Lingua tonale, il lhukonzo fa parte delle lingue bantu, ma non della famiglia del runyakitara a cui appartengono quelle, non più tonali, dei regni che hanno controllato il Rwenzori. Noto anche come konjo, nella classificazione standard appartiene alla sotto-classe J delle lingue bantu centrali (Gordon 2005).

16. In lhukonzo il termine per indicare le etnie compresenti nel territorio è olhughanda (pl. ekyaghanda), dai Bakonzo correntemente tradotto in inglese con il termine tribe (Stacey 2003).

17. Imposto dal governo britannico in seno all'Indirect Rule, il sistema elettivo statale è organizzato in cinque livelli territoriali concentrici: il distretto, la contea, la sottocontea, la parrocchia e il villaggio.

18. La denominazione stessa dei Bakonzo è passata per questa doppia mediazione: il konjo della classificazione linguistica standard, così come le diverse grafie della letteratura etnografica e degli articoli dei giornali ugandesi (lukonzo, lukonjo, rukonjo, kikonjo e lukonja), derivano dalla pronuncia del rutooro, mentre i Bakonzo prediligono la grafia qui adottata (Balinandi Kambale 2002).

19. Si narra che alla fine degli anni ' 70 , in occasione della prima trasmissione radiofonica in lhukonzo, alcuni anziani dei villaggi si sentirono così turbati e offesi da distruggere gli apparecchi che «avevano parlato lhukonzo»: attraverso la radio sarebbe dovuta arrivare la modernità, non le catene della cultura retrograda e stagnante.

20. A seguito dell'introduzione sperimentale delle lingue locali nel sistema di istruzione nazionale sono oggi disponibili testi scolastici per le classi prima, seconda e terza elementare e alcuni testi liturgici. Ad eccezione degli insegnanti elementari, però, la popolazione adulta non conosce il sistema di scrittura e di lettura del lhukonzo, standardizzato - e poi ulteriormente modificato - solo a partire dai tardi anni ' 90 .

21. Il 27 novembre 2016 le truppe governative e la polizia hanno attaccato il palazzo reale di Charles Wesley Mumbere, a Kasese. Gli scontri hanno fatto almeno un centinaio di morti, fra cui anche una quindicina di soldati. Molte delle vittime erano civili e, al termine del conflitto, oltre 180 persone, fra cui lo stesso Charles, sono state arrestate e la tensione nella regione di Kasese è andata peggiorando. Nella missione del giugno 2017 abbiamo rilevato una significativa modificazione nella percezione del regno Rwenzururu: mentre negli anni precedenti gli uomini e le donne konzo lo menzionavano spesso come orizzonte di speranza e di re-instaurazione di un modo "ecologico" di gestire le comunità e il territorio, nel 2017 il regno era menzionato in modo molto più sporadico e ambivalente.

22. Intervista a Adele K., 31/07/2010 (v. oltre la narrazione del caso).

23. Toponimo lhukonzo per l'area amministrativamente chiamata Bundibugyo: "territorio", bw-, abitato dagli Amba.

24. L'ecologia arboricola dei Batwa valse loro, all'arrivo dei primi esploratori europei, un sospetto di "scarsa umanità" e un intento civilizzatore particolarmente distruttivo. Lo stesso sospetto si ritrova nella narrazione bakonzo sulle origini della circoncisione, che i Baamba avrebbero appreso dai "più animali" Batwa; ed è rimarchevole come, oggi, l'altra caratteristica per cui i Bakonzo sono noti al resto del paese sia la presunta bassa statura che, nell'immaginario ugandese, li accomuna ai Batwa in una zona di meno certa umanità.

25. Sul valore antropopoietico dei vestiti cfr., fra l'altro, Comaroff, Comaroff 1992.

26. In kinande e in lhukonzo il prefisso eri- ha la stessa funzione del prefisso $\mathrm{ku}$-nelle altre lingue della regione e indica la forma verbale all'infinito. La radice band-, nella sua forma passiva $(+w)$, significa "essere schiacciati, oppressi da un'entità esterna" - cioè posseduti. L'eribandwa è dunque la forma della possessione spiritica kubandwa che s'incontra tra i Banande e i Bakonzo.

27. Per via del suo carattere sostanzialmente benigno, il nome di Nyamuhanga è stato utilizzato come traduzione del vocabolo cristiano "dio". Per una critica di questo genere di appropriazioni linguistiche, Singleton 2017. 
28. Un detto bakonzo afferma: «se vedi un bianco che viene da monte, scappa; se vedi un bianco che viene da valle, non c'è pericolo», con una strana commistione tra il biancore delle divinità dei ghiacciai e il pallore dei colonizzatori.

29. Mentre le famiglie della "tradizionale" Bundibugyo spesso ancora lo possiedono, altrove hanno l'endara solo gli albini.

30. Frammenti della storia di Muhindo emergevano spesso nei racconti di diversi informatori, che lo descrivevano come un caso esemplare di erisire. Il caso è stato sistematizzato grazie a diversi contatti con abitanti di Ibanda e a due interviste mirate: una prima a Muhindo, effettuata il 28/07/2010, una seconda a due suoi fratelli, effettuata in sua presenza il 30/07/2010.

31. Durante le ricerche sul campo abbiamo incontrato almeno un paio di casi ben documentati di erisire derivante dall'attribuzione al clan sbagliato, mentre abbiamo solo sentito raccontare di altri provocati dalla mancata pulizia dei crinali. Nell'eziologia Bakonzo, in sostanza, l'erisire ha sempre cause esogene (Vallarino Gancia 2006).

32. Da un'intervista all'embandwa Florence Kabugho del luglio 2010.

33. Quando il percorso terapeutico sia consolidato, il rischio che il paziente scappi si riduce e spesso è sufficiente legargli le caviglie, per consentirgli di muoversi senza allontanarsi troppo, oppure attaccargli un campanello che permetta di seguirne gli spostamenti.

34. Culture in lhukonzo, cultural things nella traduzione inglese fornita dall'informatore di campo. Vale la pena di notare che il vocabolo correntemente utilizzato in lingua lhukonzo per riferirsi alla logica locale è proprio culture, preso a prestito dall'inglese non esistendone in lhukonzo uno equivalente. Quando invece i Bakonzo parlano della logica portata dalla modernità, impiegano due termini differenti: il verbo e sostantivo erisire, visto sopra, e la radice -sungu, spesso declinata a indicare ciò che pertiene ai "bianchi", agli inglesi, all'occidente - ovvero agli abasungu (sing. musungu) che parlano lhusungu e i cui oggetti, pensieri e azioni sono kisungu.

35. Anche in questo caso, notizie sul stato di sofferenza di Mary provenivano da diverse fonti e, in particolare, da molti colloqui informali col fratello Arnold. La raccolta formale della sua storia è stata effettuata attraverso due interviste al padre (07/08/2010 e 25/08/2010), e una a Mary $(23 / 08 / 2010)$, che non ha avuto bisogno di un traduttore poiché la giovane si esprime correntemente in inglese. Inoltre, trattandosi di famiglia molto cattolica, il parroco di Ibanda è stato un utile confronto.

36. Le stesse che fra i Banande sono demandate al potere del mughula, ovvero dell'anti-capo (Remotti 1993: 216); di queste circostanze fa parte, ad es., la rimozione del cadavere di un suicida dalla capanna dove si è tolto la vita.

37. Sottolineiamo che, nelle descrizioni raccolte sul campo del complesso sistema matrimoniale konzo - che prevede il graduale trasferimento di dodici capre e di diversi oggetti definiti dalla tradizione (una zappa, una coperta di corteccia di ficus, un cesto di sementi ecc.) dalla famiglia dello sposo a quella della sposa -, la trasformazione di una parte delle capre e dei beni in somme di denaro ed elementi del corredo occidentale è correlata alla sovrapposizione con il matrimonio cristiano.

38. La sua storia è stata raccolta grazie a tre interviste: due col padre di Adele, sig. P. Mumbere (la prima, il 15/01/2010, presso la sua abitazione e in presenza della prima moglie e madre putativa di Adele; la seconda, il 20/01/2010, presso il bar dell'albergo di Ibanda); e una a Adele stessa, svoltasi a casa del padre il 31/07/2010, in presenza dapprima del padre, poi da sola.

39. Non esiste una classificazione univoca degli spiriti konzo. A differenza del contesto Banande, gli spiriti dell'omusitu sono anche le divinità del pantheon konzo; gli altri spiriti - variamente descritti come spiriti dell'acqua, della pianura, della natura - si differenziano dai primi non solo per la diversa residenza, ma anche perché non sono considerati come divinità.

40. In occasione della quale le donne della famiglia del marito organizzavano un rito a cui veniva attribuito un certo valore iniziatico: l'erikenza. Esso attestava pubblicamente il fatto che, oltre alle 
normali attività già svolte molte volte, la giovane donna era in grado di adempiere tutti i compiti femminili, inclusa la produzione della prole.

41. Come nel caso di B.K., di cui hanno dato notizia le pagine fiorentine di «Repubblica» (http:// ricerca.repubblica.it/repubblica/archivio/repubblica/2016/10/05/il-giovane-profugo-ucciso-daisuoi-fantasmiFirenze09.html).

42. Muhindo John, intervista del 28/07/2010.

43. Quando poi il cristianesimo fallisce nella risoluzione delle questioni spiritiche, la famiglia di Mumbere si avvicina all'Islam.

\section{RIASSUNTI}

Gestito in modo da occultare e naturalizzare la violenza geopolitica postcoloniale, il fenomeno migratorio, che porta il campo antropologico "sotto casa", ci mette oggi di fronte a forme di crisi inedite, sia fra i migranti che fra gli operatori. In particolare, risultano indispensabili una riflessione sulla possibilità di essere costruiti come umani da set multipli di attaccamenti; e la capacità di lavorare i presupposti impliciti, i non detti, i rimossi. I Bakonzo del Rwenzori (Uganda), attualmente alle prese con le intersezioni fra la logica antropopoietica locale e quella imposta dalla modernità euro-americana, e con le crisi che talora ne scaturiscono, sono un eccellente caso di studio, che permette una triangolazione analogica con quanto avviene qui. Non si tratta di confrontare contenuti, ma di osservare le dinamiche in azione nella pluralità: se ne ricava che la molteplicità non è di per sé fattore di crisi e, anzi, agisce tanto nella vita ordinaria quanto nella crisi come un moltiplicatore di possibilità. La compresenza di diversi insiemi di attaccamenti diventa semmai problematica quando uno degli insiemi di attaccamenti pretende di essere esclusivo.

Managed in a way that both conceals and naturalizes postcolonial geopolitical violence, current migratory flows bring the anthropological field in our backyards, facing us with unprecedented forms of crisis both among migrants and among operators. It seems especially necessary to consider the actual possibility for persons to be built by means of multiple sets of attachments; and the ability to work implicit presuppositions, un-told and repressed events. The Bakonzo of the Rwenzori (Uganda), who are facing the intersection between the local anthropo-poietic logic and the one brought about by Euro-American "modernity", make for and an excellent case study, allowing for an analogical comparison with what is happening here. The point is not to compare contents, but to be able to observe those dynamics that emerge in plural contexts. It may be concluded that multiplicity is not a risk factor in itself, rather, it acts as a multiplier of possibilities both in ordinary life and in critical times. The simultaneous presence of various sets of attachments proves problematic if one of the sets demands exclusivity.

\section{INDICE}

Parole chiave : Bakonzo, antropopoiesi, attaccamenti, molteplicità, crisi

Keywords : Bakonzo, anthropo-poiesis, attachments, multiplicity, crisis 


\section{AUTORI}

\section{STEFANIA CONSIGLIERE}

Dipartimento di Scienze della Formazione, Università degli Studi di Genova stefania.consigliere[at]unige.it

\section{CRISTINA ZAVARONI}

Università degli Studi di Torino cristina.zavaroni[at]unito.it 